\title{
A LARGE-AREA SEARCH FOR LOW-MASS OBJECTS IN UPPER SCORPIUS. I. THE PHOTOMETRIC CAMPAIGN AND NEW BROWN DWARFS
}

\author{
Catherine L. Slesnick, John M. Carpenter, and Lynne A. Hillenbrand \\ Department of Astronomy, California Institute of Technology, MC 105-24, Pasadena, CA 91125; \\ cls@astro.caltech.edu,jmc@astro.caltech.edu, lah@astro.caltech.edu \\ Received 2005 December 24; accepted 2006 February 14
}

\begin{abstract}
We present a wide-field photometric survey covering $\sim 200 \mathrm{deg}^{2}$ toward the Upper Scorpius OB association. Data taken in the $R$ and $I$ bands with the Quest- 2 camera on the Palomar 48 inch $(1.2 \mathrm{~m})$ telescope were combined with the Two Micron All Sky Survey $J H K_{S}$ survey and used to select candidate pre-main-sequence stars. Follow-up spectroscopy with the Palomar 200 inch $(5.1 \mathrm{~m})$ telescope of 62 candidate late-type members identified 43 stars that have surface gravity signatures consistent with association membership. From the optical/near-infrared photometry and derived spectral types we construct an H-R diagram for the new members and find 30 likely new brown dwarfs, nearly doubling the known substellar population of the Upper Scorpius OB association. Continuation of our spectroscopic campaign should reveal hundreds of new stellar and substellar members.
\end{abstract}

Key words: open clusters and associations: individual (Upper Scorpius) - stars: low-mass, brown dwarfs stars: pre-main-sequence

\section{INTRODUCTION}

The Upper Scorpius OB Association (USco) is the closest (145 pc; de Zeeuw et al. 1999) young OB association to the Sun, with 114 known high-mass Hipparcos stars. At an age of $\sim 5 \mathrm{Myr}$ (Preibisch et al. 2002), this cluster is at an intermediate age between very young star-forming regions and older open clusters for where samples are sparser and studies of processes such as circumstellar disk dissipation are critical. Recent mid-infrared work by Mamajek et al. (2004) and Silverstone et al. (2006) indicates that by $\sim 10 \mathrm{Myr}$ dust is removed from the inner few AU of circumstellar disks for $\gtrsim 85 \%$ of stars, whereas $\sim 80 \%$ of young $1 \mathrm{Myr}$ stars in Taurus still retain their disks at these radii (Kenyon \& Hartmann 1995; Skrutskie et al. 1990). This evolution in circumstellar material corresponds to the stage when planets are thought to be forming. Meteoritic evidence suggests that the timescale for the dissipation of our own solar system's nebula was on the order of $10^{7} \mathrm{yr}$ (Podosek \& Cassen 1994). Furthermore, discovery of ${ }^{60} \mathrm{Fe}$ in meteorites argues that short-lived radionuclides were injected into the solar system's early protoplanetary disk from the explosion of a nearby supernova (Desch \& Ouellette 2005; Tachibana \& Huss 2003). The evidence strongly suggests that our solar system was formed in an $\mathrm{OB}$ association similar to USco. Therefore, if we are to understand our own Earth's origins, we must study the evolution of $\mathrm{OB}$ association members during planet-building stages.

A major difficulty faced by studies of the USco region is that the Hipparcos members alone span $>130 \mathrm{deg}^{2}$ on the sky. Obtaining a complete census of the association's low-mass population is thus a formidable task, as one must identify faint objects over a very large spatial region. While there exist several techniques to identify young stars not associated with molecular gas, many of them are also accretion diagnostics. For example, a common method is to search for strong $\mathrm{H} \alpha$ emission (Ardila et al. 2000 ) produced in outflow or accretion flows (see $\S 3.4$ ), or nearinfrared excess emission associated with warm inner accretion disks. While accretion can terminate over a wide range in age (1$10 \mathrm{Myr}$ ), the median lifetime of optically thick accretion disks is closer to 2-3 Myr (Haisch et al. 2001; Hillenbrand 2006). Therefore, surveys to look for accretion signatures alone will not garner a full census of a $5 \mathrm{Myr}$ association.
Enhanced chromospheric and coronal activity can last well beyond accretion timescales. This activity is linked with X-ray emission (Ku \& Chanan 1979; Feigelson \& Decampli 1981), although the exact cause of this phenomenon is still not fully understood (Preibisch et al. 2005). Many previous large-scale efforts in USco have successfully used Einstein Observatory data (Walter et al. 1994) or the ROSAT All Sky Survey (RASS; Preibisch et al. 1998; Preibisch \& Zinnecker 1999) to identify hundreds of lowand intermediate-mass association members. However, neither the Einstein observations nor the RASS were sensitive enough to detect faint X-ray emission from the lowest mass stars and brown dwarfs.

Recently, deep multicolor imaging surveys combined with spectroscopic follow-up have proved successful in identifying both the youngest classical T Tauri-type objects and more evolved very low mass stars and brown dwarfs in a variety of young regions. Young pre-main-sequence (PMS) objects still undergoing contraction toward the main sequence are redder and more luminous than their main-sequence counterparts. Spectroscopic followup observations allow assessment of surface gravity diagnostics that can be used to distinguish young PMS stars from reddened field dwarfs and background giants. Previous imaging and spectroscopic surveys in USco include work by Preibisch et al. (2001, 2002), who selected candidate association members based on optical magnitudes and colors obtained from photographic plates in the United Kingdom Schmidt Telescope survey fields. Their spectroscopic survey of 700 candidates over $9 \mathrm{deg}^{2}$ using the $2 \mathrm{dF}$ multifiber spectrograph yielded 166 new PMS stars based on the presence of lithium in their spectra. Martín et al. (2004) selected candidate young objects from the Deep Near Infrared Survey of the Southern Sky (DENIS) $I, J$ survey and obtained spectra of 36 targets. Of these, 28 were confirmed to be new low-mass association members based on surface-gravity diagnostics. Ardila et al. (2000) used $R, I, Z$ photographic photometry to identify candidate members within eight $80^{\prime} \times 80^{\prime}$ areas of the association. Spectroscopic data were obtained for 22 candidates, 20 of which were determined to exhibit $\mathrm{H} \alpha$ emission, indicating possible membership. Thus far, over 300 low-mass $\left(M<0.6 M_{\odot}\right)$ members have been identified in USco through X-rays, $\mathrm{H} \alpha$ emission, photometry, and/or spectroscopy. However, most searches have been limited 


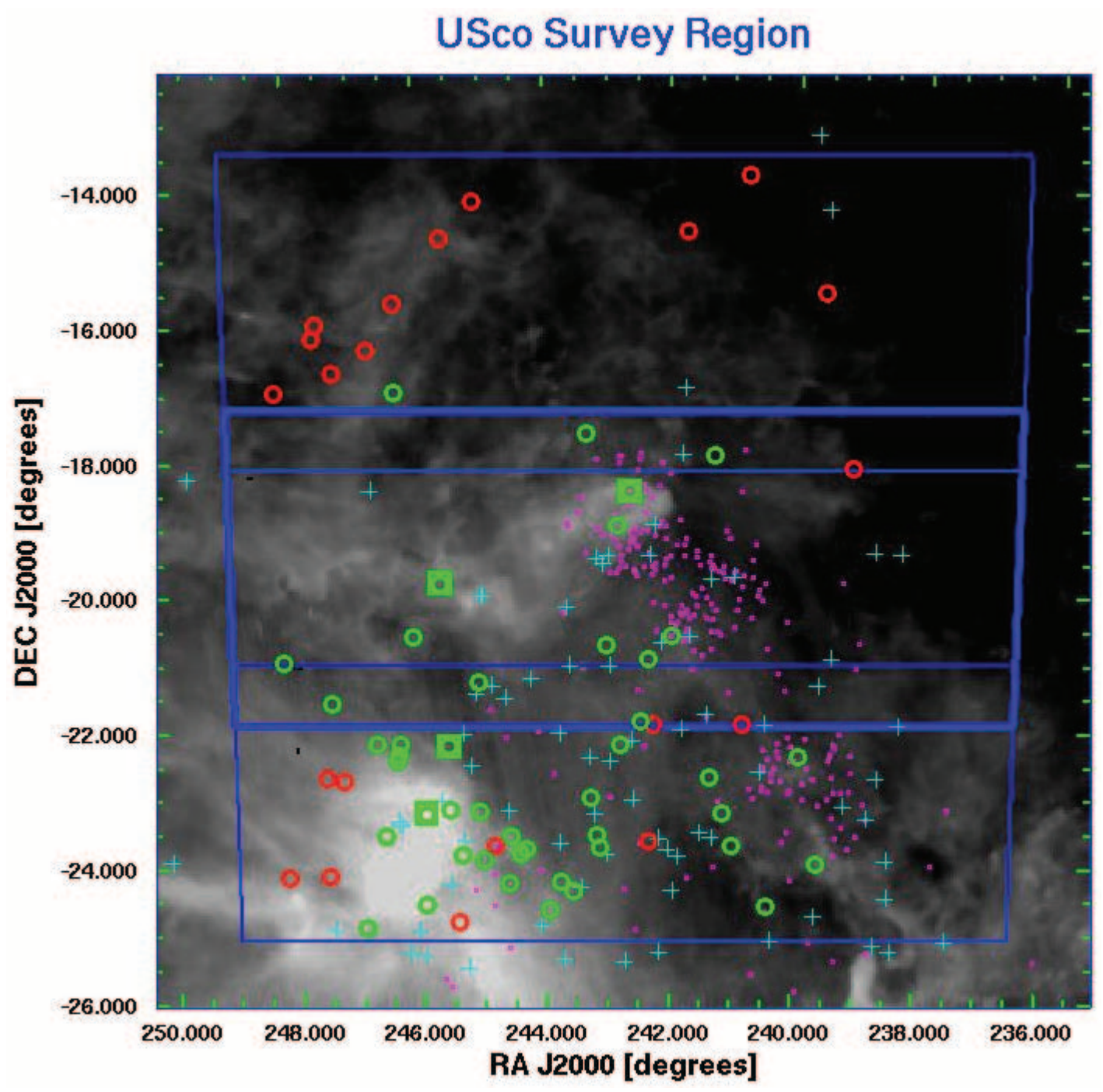

FIG. 1.-Spatial distribution of the USco survey area outlined in blue, overlaid on IRAS $100 \mu \mathrm{m}$ emission. The thicker blue line denotes the outline of the monitoring scan region, which was repeated 24 times. New USco members identified from this work (43; green circles) are shown with previously known, spectroscopically confirmed low-mass members (196; spectral type $\geq \mathrm{K} 7$, corresponding to $M \leq 0.6 M_{\odot}$ at $5 \mathrm{Myr}$; magenta circles) from the literature (see text), high-mass Hipparcos members (114; cyan plus signs), and spectroscopic targets determined to be field dwarfs (22; red circles). New members that exhibit very strong H $\alpha$ emission are boxed.

to small subregions or bright objects. Given the USco upper initial mass function (IMF), and assuming the high- and low-mass objects share the same spatial distribution, Preibisch et al. (2002) estimate that the entire USco region should contain $>1500$ young low-mass objects with $M<0.6 M_{\odot}$, most of which are yet to be discovered.

Building on previous work in this region, we have completed a large-area optical photometric survey of $\approx 200 \mathrm{deg}^{2}$ in and near USco. We combine these data $(\S 2)$ with the Two Micron All Sky Survey (2MASS) $J, H, K_{S}$ photometry to select candidate young PMS objects. In $\S 3$ we present newly obtained optical spectra for 62 candidate young brown dwarfs and low-mass stars. These spectra allow us to determine spectral type and confirm membership for photometrically selected candidates. Finally, in $\S 4$ we derive an $\mathrm{H}-\mathrm{R}$ diagram and discuss noteworthy new members.

\section{OBSERVATIONS}

\subsection{Photometric Monitoring and Data Processing}

Photometric observations were taken with the Quest-2 camera (Rabinowitz et al. 2003) on the 48 inch (1.2 m) Samuel
Oschin Schmidt Telescope at Palomar Observatory. The Quest-2 camera is a large-area mosaic of 112 CCDs arranged in a grid of 4 columns by 28 rows. Each CCD has $600 \times 2400$ pixels with a scale of $\sim 0^{\prime \prime} .8$ pixel $^{-1}$. The camera covers a $3^{\circ} .6 \times 4^{\circ} .6$ field of view. Taking into account gaps between columns and chips the total sky coverage is $9.4 \mathrm{deg}^{2}$ per pointing. Each of the four columns views the sky through a separate filter.

Data were obtained in drift-scan format using $U, B, R, I$ filters. Any given patch of sky is observed over the entire 2400 pixel width of four separate CCDs (one per filter) in one of the 28 rows. Charge is continuously read out of each CCD throughout the observation, and the final data product is a strip of uniform width in declination and time-dependent length in right ascension. Three scans, centered at $\delta=-15^{\circ} .7,-19^{\circ} .5$, and $-23^{\circ} .3$, were each observed between R.A. of $15^{\mathrm{h}} 46^{\mathrm{m}}$ and $16^{\mathrm{h}} 36^{\mathrm{m}}$. The scan centered at $\delta=-19.5$ was observed three to four times per night on seven consecutive photometric nights over 2004 June 20-26. The other two scans were observed once during this period. Spatial coverage is shown in Figure 1. Because several CCDs have failed since installation due to bad connections or faulty chips, our spatial coverage within the survey region is not uniform. Five of 28 CCDs in 
the $I$ column, five in the $R$ column, and four in the $B$ column are not fully functional. The CCDs are less sensitive in the $U$ band than anticipated, and very few source detections were obtained. As discussed in $\S 2.3$, we are primarily concerned with selecting faint red objects as candidate young low-mass stars, the majority of which do not have a $B$-band detection. We therefore exclude the $U$ and $B$ data from the remainder of this discussion.

When operated in drift-scan format, the Quest- 2 camera generates $\approx 50 \mathrm{~GB}$ of raw, compressed data in a single night. To efficiently process such a large volume of data, the Quest-2 Collaboration (Rabinowitz et al. 2003) has developed automated data reduction software (Andrews 2003). Each of the CCDs is treated as a separate instrument during reductions, and therefore, we explain the procedure for a single chip. The software first performs the basic bias subtraction and flat-fielding. The bias level for a given column of the CCD is computed by median-combining 25 rows in the overscan region and is then subtracted. Dark subtraction and flat-fielding cannot be carried out using the standard techniques of pointed observations. Instead, sky-flat and dark drift scans were taken on the first night of observations. Each calibration image was first divided into $240600 \times 10$ pixel segments and then median-combined using the IRAF imsum task to remove cosmic rays (darks) or stars (sky flats). The resulting $600 \times 10$ pixel image was then averaged into a single row of 600 pixels using the IRAF blkavg task. The averaged dark and flat rows were subtracted and divided, respectively, from each row of data.

After preprocessing, the pipeline generates a point-spread function $(\mathrm{PSF})$ for every frame $(600 \times 2000$ pixels $)$ of data, which it uses to detect sources. It registers detections from all four filters and generates an astrometric solution from the USNO-A2 catalog. We have matched detections within a 0.8 radius from up to 24 different scans in the monitoring region. For each source we computed new coordinates by averaging together coordinates from individual scans and found typical astrometric residuals about the mean for individual detections to be \pm 0 "' 1 .

The Yale pipeline generates photometry both from a range of apertures and from PSF function fitting. We compared PSF and aperture photometry and found that the aperture photometry produced more consistent results for sources observed in multiple scans (see $\S 2.2$ for a detailed discussion of photometric reliability). We therefore chose to rely on aperture photometry despite the potential advantages of PSF fitting for crowded stars. The pipeline measures all objects through an aperture of half-width of 3.5 pixels (found to produce the highest signal-to-noise ratio $[\mathrm{S} / \mathrm{N}]$ photometry for a subset of bright isolated stars). To account for zero-point variations between chips we applied the calibrations derived by the Quest-2 instrument group at Yale (J. Jerke et al. 2005, private communication). These calibrations also converted Quest-2 instrumental magnitudes into the Johnson photometric system.

We accounted for small atmospheric transparency changes during the night and between nights by applying a photometric offset to every scan as a function of right ascension. We selected a subset of calibrator stars from our source catalog that were detected in at least 20 out of 24 of the $\delta=-19^{\circ} .5$ monitoring scans and had no neighbors within $5^{\prime \prime}$. For each star and filter an uncertainty-weighted average magnitude of all detections was calculated along with the difference between that average magnitude and the magnitude measured on each individual scan. For every chip we created a catalog of offsets by stepping through in right ascension every 25 calibrator stars and calculating a median offset value. We applied these offsets of up to $0.25 \mathrm{mag}$ to the entire data set as a function of right ascension and CCD chip by linearly interpolating in right ascension values. For the high

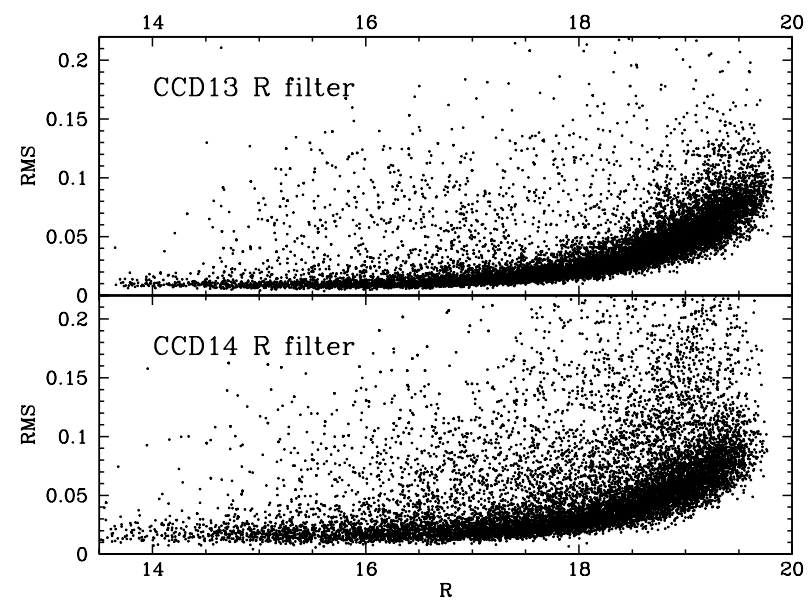

FIG. 2.-Computed rms deviations as a function of magnitude for two CCDs. Each magnitude is the average of 10-24 measurements, and the corresponding rms is a weighted deviation of all individual measurements about that average. The top and bottom panels show, respectively, repeatability plots for a "best" and a typical CCD. See text for further explanation.

$\left(\delta=-15^{\circ} .7\right)$ and low $\left(\delta=-23^{\circ} .3\right)$ declination scans that overlap only the top and bottom 0.8 of the monitoring scan region, we calculated a scan offset as a function of right ascension using the same procedure outlined above, but averaging together all columns of overlap with the mid-declination scans, rather than chip by chip. We found that this procedure produced magnitudes consistent with those derived from the multiple-scan region at the $7 \%(R)$ and $2 \%(I)$ levels for objects in the overlap region. Finally, to account for differences in air mass between the Yale calibrator scans and USco scans we applied offsets of 0.08 and 0.05 mag for the $R$ and $I$ data, respectively (Parrao \& Schuster 2003) as derived at San Pedro Mártir and consistent with typical Palomar extinction coefficients.

\subsection{Precision and Accuracy}

In this section we use photometry from the monitoring scans to assess the relative precision of the photometric data. We find photometric precision to be CCD dependent and give values for best, worst, and typical scenarios. The photometric accuracy is harder to quantify at this time. Zero points for individual CCDs and conversions from Quest-2 to Johnson photometry have not yet been finalized by the Quest-2/Yale instrument team. To place some constraints on data accuracy we have matched the $R$ - and $I$-band photometry to photometry from Ardila et al. (2000) and have examined the consistency.

For each source in the multiple-scan region, an uncertaintyweighted average and the rms deviation of the individual measurements were calculated. The 112 CCDs are of varying quality, which leads to nonuniform photometric characteristics. Figure 2 shows the rms as a function of magnitude for a single CCD for sources detected at least 10 times. The top panel shows data for the CCD in row 13, column 1 ( $R$ filter), one of the most reliable CCDs. The bottom panel shows data for an adjacent CCD in row 14, column 1, representative of a typical CCD. The best CCDs have rms values from $\sim 0.01 \mathrm{mag}$ for the bright stars and to $\sim 0.05 \mathrm{mag}$ by $R=19$ and $I=18.5$. A typical CCD has rms $\sim 0.03 \mathrm{mag}$ at the bright end to $\sim 0.08 \mathrm{mag}$ at the faint end. We find six CCDs to be of poor quality, with rms ranging from $\sim 0.07$ to 0.15 mag at the bright and faint ends.

The accuracy of the photometric transformations from the Quest-2 to the Johnson system is harder to quantify since 


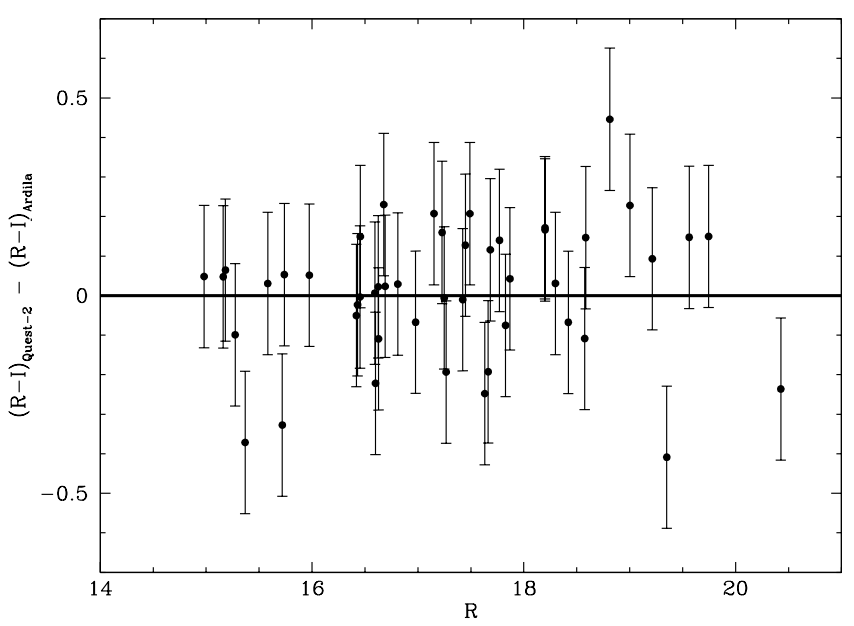

Fig. 3.-Difference between Quest-2 $R-I$ colors and $R-I$ colors from Ardila et al. (2000) (converted to the Johnson system) as a function of Quest-2 $R$ magnitude. Error bars reflect rss uncertainties in both data sets.

differences both in zero points between chips and in conversion between photometric systems must be accounted for. The Quest-2 instrument team at Yale has matched Quest-2 observations in the north to Sloan Digital Sky Survey (SDSS) photometry and derived conversions for each chip from Quest-2 to SDSS magnitudes (converted to the Johnson system; Fukugita et al. 1996). We have used the current calibrations and attempted to quantify the accuracy of the photometry presented here by comparing our data to those of Ardila et al. (2000), who observed $\sim 14 \mathrm{deg}^{2}$ in USco in the Cousins $R$ and $I$ bands. Figure 3 shows the difference between Quest-2 Johnson $R-I$ colors and those from Ardila et al. (2000) converted from the Cousins to the Johnson system (Fernie 1983) as a function of Quest-2 Johnson $R$ magnitude. Error bars of \pm 0.18 mag reflect average root-sum-squared (rss) uncertainties from both data sets: $\left\langle\sigma_{R-I}\right\rangle=0.14$ in the Ardila et al. (2000) photometry (D. Ardila 2005, private communication) and $\left\langle\sigma_{R-I}\right\rangle=$ 0.11 for the Quest-2 photometry assuming typical magnitude uncertainties of $0.08 \mathrm{mag}$ for faint stars. The average difference between the two data sets is 0.01 mag with an rms deviation of 0.17 , consistent with uncertainties.

From histograms of all detections for the $R$ and $I$ filters we find a turnover in number of objects detected at $R \sim 19$ mag and $I \sim$ 18 mag, with a substantial number of detections out to $R \sim 20$ and $I \sim 19$. We believe the precision of our photometry to be $\lesssim 0.08$ mag for $\sim 90 \%$ of stars brighter than these values. Both $R$ and $I$ data saturate for stars brighter than $\sim 13$ mag.

\subsection{Candidate Member Selection}

The detection algorithm in the Yale data pipeline was written to find very faint quasars and therefore uses a low detection threshold. To produce a reliable catalog of sources, we first required an object to be detected in both the $R$ and $I$ filters. Furthermore, we matched our entire data set to the 2MASS All Sky Catalog (Cutri et al. 2003) and considered stars as potential candidates only if they had a 2 MASS counterpart within $2^{\prime \prime}$. This cut biases our final catalog against faint blue objects, to which 2MASS is not sensitive. Of the $\sim 1.5$ million objects in our source catalog, $\sim 500,000$ have 2 MASS counterparts. However, because we are interested only in objects redder than a $30 \mathrm{Myr}$ isochrone in an optical colormagnitude diagram (CMD; see below for details) and bright enough to be observed spectroscopically ( $R \lesssim 20 \mathrm{mag}$ ), this bias does not affect candidate selection in practice. Figure 4 shows contour optical CMDs of all Quest-2 sources (black) and those

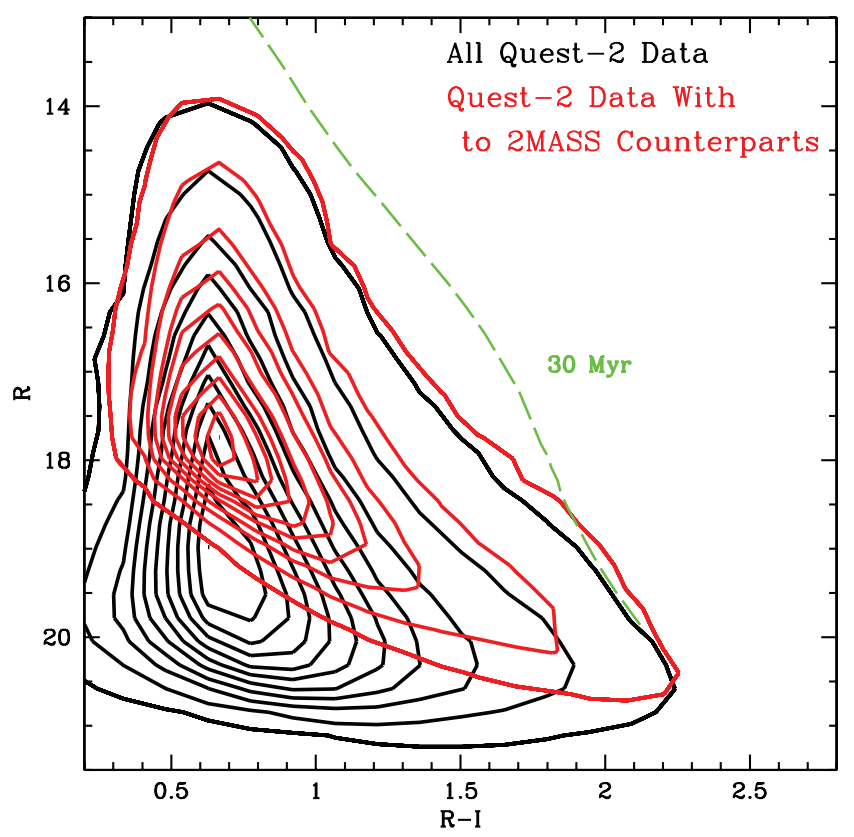

FIG. 4.-Optical CMDs of all Quest-2 sources (black) and those with 2MASS detections (red). For both black and red contours, data are represented from $90 \%$ to $10 \%$ and at $3 \%$ of the peak level.

with 2MASS detections (red). As can be seen, the red side of the CMD brighter than $R \sim 20$ is not substantially affected by excluding objects without a 2 MASS detection.

The final photometric catalog that we use to select candidate association members contains $\sim 500,000$ sources with $R, I, J, H$, and $K_{S}$ detections, most of which are field stars. However, buried in these $\sim 500,000$ sources are several thousand bona fide lowmass members of USco. We use this data set to select candidate PMS stars based on an optical CMD indicating that they could be young USco members. In Figures 5-7 we present color-magnitude

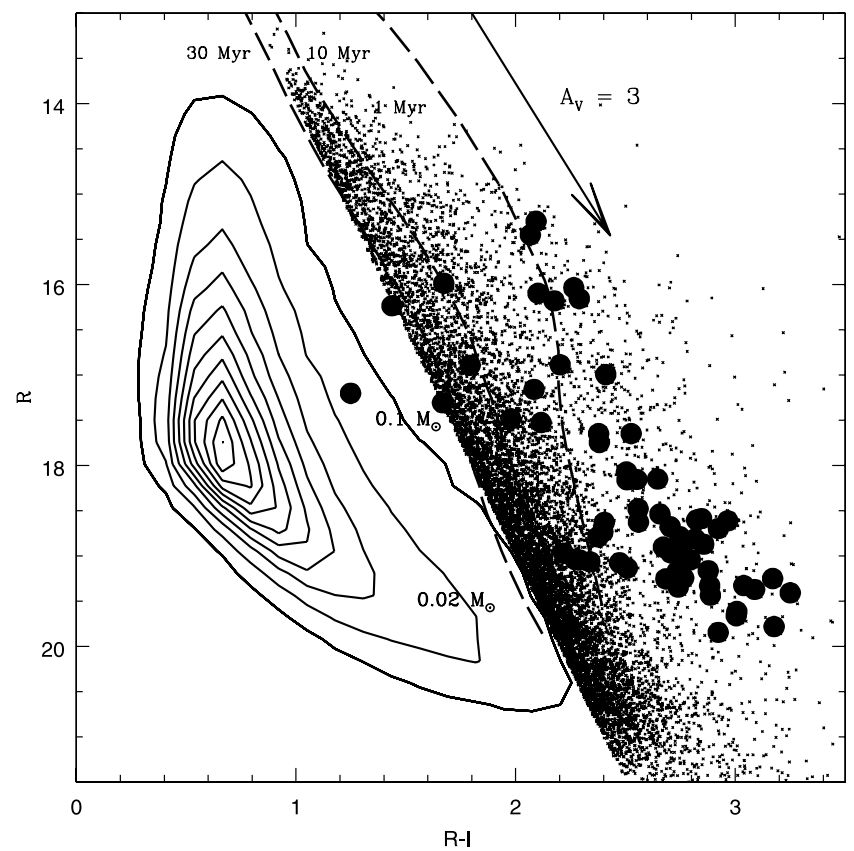

FIG. 5. $-R, R-I$ CMD for all sources with a 2 MASS detection, shown with isochrones from DM97 transformed by us into the $(R, R-I)$ plane. Contours are as described in Fig. 4. Objects that appear younger than $\sim 30 \mathrm{Myr}$ are shown as discrete points. Spectroscopic targets presented here are shown as large circles. 


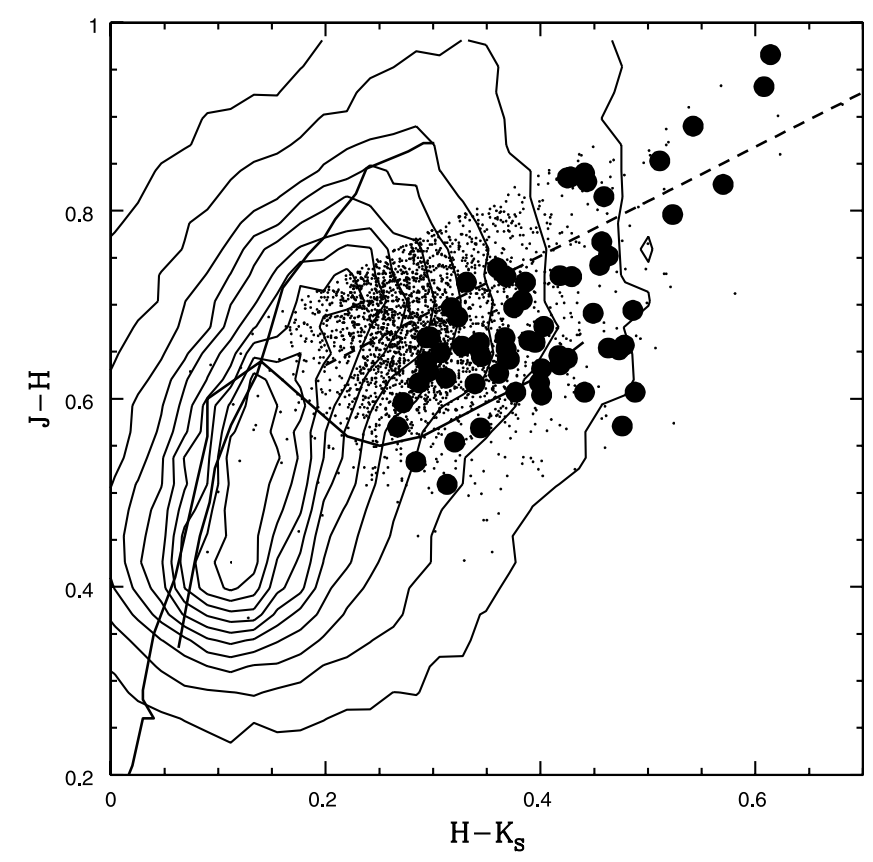

Fig. 6.-2MASS $J-H, H-K_{S}$ color-color diagram for all data, represented as contours from $90 \%$ to $10 \%$ and at $3 \%$ of the peak level. Shown as discrete points are all objects that appear younger than $30 \mathrm{Myr}$ in an optical $\mathrm{CMD}$ and do not have colors consistent with those of background giants. Solid lines show dwarf zero-age main sequence (O5-M8) and giant loci (G0M7). The dashed line represents the classical T Tauri locus as defined by Meyer et al. (1997). Spectroscopic targets presented here are shown as large circles.

and color-color diagrams used in PMS candidate selection. For the work presented here, we used three levels of selection criteria. In each figure, objects that meet the outlined selection criteria and all previous selection criteria are shown as discreet points. In Figure 5 we show the $R, R-I \mathrm{CMD}$ for all sources matched to 2MASS. About 60,000 sources with $R<5(R-I-0.4)+11$ appear younger than $\sim 30 \mathrm{Myr}$ based on theoretical isochrones (D'Antona \& Mazzitelli 1997, hereafter DM97; 1998) ${ }^{1}$ and could be PMS objects.

Photometry from 2MASS photometry was used to further refine the candidate sample. In Figure 6 we show a near-infrared color-color diagram. Solid lines represent the dwarf (bottom line) and giant (top line) loci. The dashed line represents the classical T Tauri star locus as defined by Meyer et al. (1997). Beyond this point we considered only objects that had "good" 2 MASS photometry (quality flag "A") in all three $J, H$, and $K_{S}$ bands. Any star with $J-H, H-K_{S}$ colors consistent with those expected for background giants $\left.\left[(J-H)>0.6\left(H-K_{S}\right)+0.6\right)\right]$ was excluded.

After applying the above selection criteria we identified $\sim 20,000$ candidate new young USco members over $\sim 200 \mathrm{deg}^{2}$. Due to interstellar extinction and distance effects, optical and near-infrared colors and magnitudes alone are not a unique indicator of youth; therefore, it is necessary to obtain spectroscopic follow-up observations to determine an object's spectral type and confirm low surface gravity consistent with that of young association members. We began a spectroscopic follow-up program with five observing nights in 2005 June using the Double Spectrograph on the Palomar 200 inch $(5.1 \mathrm{~m})$ telescope and five more in 2005 July using Hydra on the Cerro Tololo Inter-American Observatory $4 \mathrm{~m}$ telescope. With the Palomar spectroscopic observa-

\footnotetext{
${ }^{1}$ The authors' 1998 models are a Web-only correction at $<0.2 M_{\odot}$ to their original 1997 work.
}

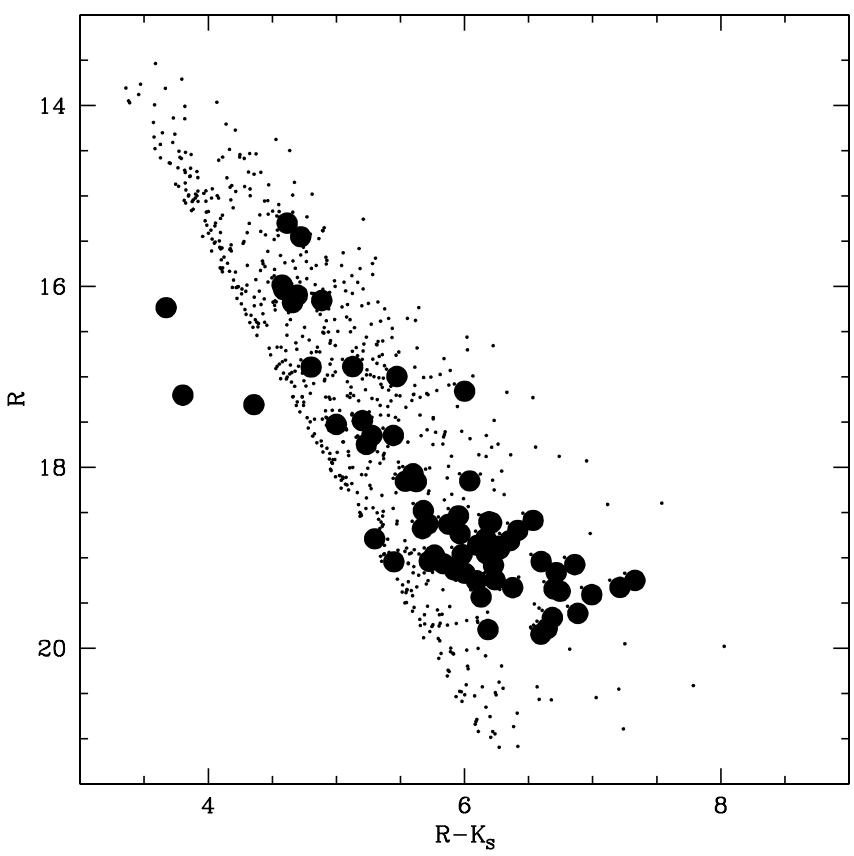

FIG. 7. $-R, R-K_{S}$ CMD for the $\sim 1000$ objects that meet the optical and near-infrared selection criteria shown in Figs. 5 and 6 and that appear red in $R-K$. Spectroscopic targets presented here are shown as large circles.

tions discussed here, we chose to target potential new brown dwarfs and very low mass stars to take full advantage of the high sensitivity of the Double Spectrograph. We therefore selected only the reddest candidates satisfying $R<2.57\left(R-K_{S}\right)+13$ in an $R, R-K_{S} \mathrm{CMD}$ (Fig. 7) and focused primarily on the faintest $(R>18.5)$ targets. With the Hydra CTIO Hydra we took spectra of close to 1000 brighter $(15<R<18.5)$ candidates, leading to new higher mass (spectral type G-M) USco members, which will be discussed in a forthcoming paper. After applying this last selection criterion we were left with $\sim 1000$ candidates suitable for spectroscopic follow-up with the Double Spectrograph, $\sim 200$ of which are fainter than $R=18.5$.

\subsection{Optical Spectroscopy}

Moderate-resolution spectra of 65 objects (Table 1; Figures 5-7, large circles) were obtained during the nights of 2005 June $8-12$ using the Double Spectrograph on the 200 inch Palomar telescope. Of these, 41 were fainter than $R \sim 18.5$, corresponding to $\sim 20 \%$ of the faint candidates meeting all of the selection criteria detailed in $\S 2.3$. Because Quest- 2 is a new instrument and software is continuously being updated, the photometric data have been re-reduced several times. Photometry for three of the spectral targets has changed significantly since the spectroscopic observations were taken, such that they are no longer considered candidates. As expected, all three were determined to be field dwarfs based on their spectra. For completeness these three sources are shown in the figures and listed in Table 1, but otherwise are not discussed further.

Data were taken using the red side of the Double Spectrograph, which has a $1024 \times 1024 \mathrm{CCD}$ with $24 \mu \mathrm{m}$ pixels. We used a 2 ". 0 entrance slit, $5500 \AA$ dichroic and 316 lines $\mathrm{mm}^{-1}$ grating blazed at $7500 \AA$, which gave a wavelength coverage of $6300-8825 \AA$ at resolution $R \sim 1250$. Typical exposure times were $600-900 \mathrm{~s}$, and we were able to observe objects as faint as $R \sim 20$ in $1800 \mathrm{~s}$ with $\mathrm{S} / \mathrm{N} \sim 30-40$. Spectrophotometric standard stars (Massey et al. 1988) were observed throughout every night for flux calibration. 
TABLE 1

Measured Quantities for PMS Candidates with Observed Spectra

\begin{tabular}{|c|c|c|c|c|c|c|c|c|c|c|c|}
\hline ID & $R$ & $I$ & $J^{\mathrm{a}}$ & $H^{\mathrm{a}}$ & $K_{S}^{\mathrm{a}}$ & TiO-7140 & TiO-8465 & Na-8189 & Spectral Type ${ }^{\mathrm{b}}$ & $\begin{array}{c}W(\mathrm{H} \alpha) \\
(\AA)\end{array}$ & Gravity $^{\mathrm{c}}$ \\
\hline 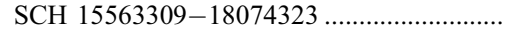 & 19.7 & 16.5 & 14.18 & 13.61 & 13.13 & 3.22 & 1.80 & 0.81 & M7 & & Dwarf \\
\hline SCH $15582384-15310335 \ldots \ldots \ldots \ldots \ldots \ldots \ldots \ldots$ & 16.1 & 13.9 & 12.36 & 11.79 & 11.52 & 1.76 & 1.16 & 0.83 & M4.5 & -5.40 & Dwarf \\
\hline 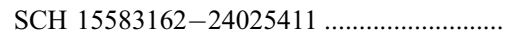 & 17.5 & 15.0 & 13.16 & 12.51 & 12.20 & 1.95 & 1.25 & 0.93 & M4.5 & -7.60 & USco \\
\hline 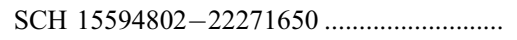 & 19.1 & 16.5 & 14.24 & 13.56 & 13.16 & 2.78 & 1.90 & 0.93 & M7.5 & -15.20 & USco \\
\hline 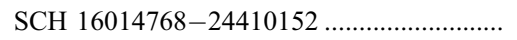 & 18.5 & 15.9 & 13.87 & 13.27 & 13.00 & 2.44 & 1.45 & 0.86 & M5 & -16.00 & USco \\
\hline SCH 16031129-13454481 … & 17.2 & 15.5 & 13.96 & 13.35 & 12.95 & 2.07 & 1.33 & 0.80 & M5 & -8.70 & Dwarf \\
\hline SCH $16032871-21583609^{\mathrm{d}} \ldots \ldots \ldots \ldots \ldots \ldots \ldots \ldots$ & 18.9 & 16.7 & 14.62 & 13.96 & 13.59 & 0.99 & 1.08 & 0.93 & K3: & $\ldots$ & Dwarf \\
\hline 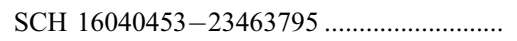 & 15.3 & 13.3 & 11.74 & 11.04 & 10.73 & 1.81 & 1.20 & 0.93 & M4 & -4.00 & Int \\
\hline 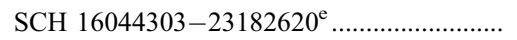 & 19.0 & 15.9 & 13.81 & 13.19 & 12.85 & 2.72 & 1.79 & 0.90 & M6.5 & -18.00 & USco \\
\hline 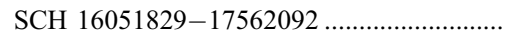 & 15.2 & 13.1 & 11.64 & 10.98 & 10.68 & 1.66 & 1.11 & 0.93 & M4 & -5.40 & Int \\
\hline 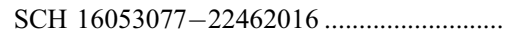 & 18.8 & 16.1 & 13.78 & 13.18 & 12.78 & 2.84 & 1.65 & 0.89 & M6 & -17.80 & USco \\
\hline 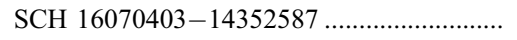 & 15.9 & 14.2 & 12.28 & 11.73 & 11.41 & 1.89 & 1.17 & 0.84 & M4 & $\ldots$ & Dwarf \\
\hline 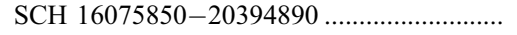 & 18.4 & 15.8 & 13.59 & 12.95 & 12.58 & 2.47 & 1.53 & 0.89 & M6 & -14.90 & USco \\
\hline 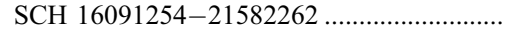 & 18.7 & 16.3 & 14.39 & 13.77 & 13.49 & 2.20 & 1.33 & 0.82 & M5 & $\ldots$ & Dwarf \\
\hline SCH $16092940-23431209 .$. & 19.0 & 16.5 & 14.20 & 13.57 & 13.21 & 0.96 & 0.96 & 1.00 & $<\mathrm{K} 3$ : & $\ldots$ & Dwarf \\
\hline 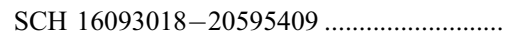 & 18.8 & 16.2 & 13.99 & 13.35 & 12.98 & 2.59 & 1.53 & 0.89 & M6 & -11.10 & USco \\
\hline 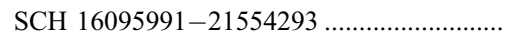 & 19.3 & 16.4 & 14.30 & 13.64 & 13.30 & 2.49 & 1.57 & 0.89 & M6.5 & -17.40 & USco \\
\hline 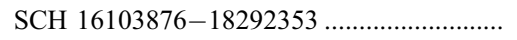 & 19.2 & 16.5 & 13.96 & 13.16 & 12.64 & 2.29 & 1.57 & 0.96 & M6 & -80.30 & USco \\
\hline 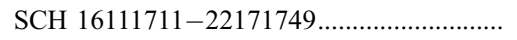 & 19.7 & 16.8 & 14.34 & 13.73 & 13.25 & 2.68 & 1.82 & 0.94 & M7.5 & -20.80 & USco \\
\hline 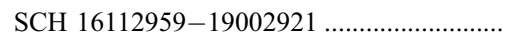 & 19.0 & 16.2 & 13.67 & 12.90 & 12.44 & 2.49 & 1.68 & 0.94 & M6 & -20.40 & USco \\
\hline 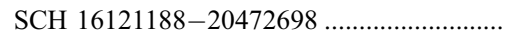 & 18.7 & 15.9 & 13.66 & 13.02 & 12.60 & 2.69 & 1.77 & 0.93 & M6.5 & -8.10 & USco \\
\hline 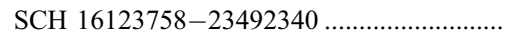 & 18.5 & 16.0 & 13.93 & 13.28 & 12.91 & 2.47 & 1.51 & 0.87 & M6 & -15.80 & USco \\
\hline 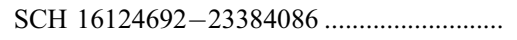 & 18.7 & 15.9 & 13.65 & 13.02 & 12.62 & 2.66 & 1.70 & 0.90 & M6 & -14.70 & USco \\
\hline 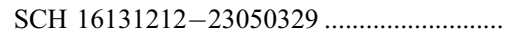 & 19.1 & 16.4 & 14.05 & 13.44 & 13.00 & 2.62 & 1.73 & 0.90 & M6.5 & -13.40 & USco \\
\hline 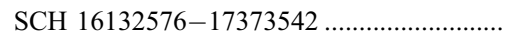 & 16.0 & 13.9 & 12.32 & 11.69 & 11.40 & 1.72 & 1.14 & 0.91 & M4 & -5.30 & Int \\
\hline 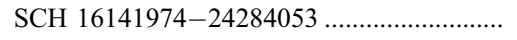 & 18.7 & 16.0 & 13.81 & 13.15 & 12.76 & 2.59 & 1.61 & 0.88 & M6 & -16.40 & USco \\
\hline 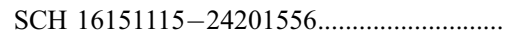 & 19.0 & 16.3 & 14.23 & 13.58 & 13.17 & 2.33 & 1.43 & 0.90 & M6 & -10.90 & USco \\
\hline 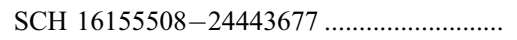 & 18.6 & 15.7 & 13.39 & 12.74 & 12.28 & 2.48 & 1.66 & 0.93 & M6 & -15.80 & USco \\
\hline 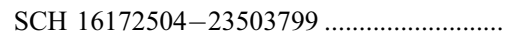 & 18.8 & 16.1 & 13.74 & 13.01 & 12.63 & 2.25 & 1.44 & 0.87 & M5 & -12.00 & USco \\
\hline 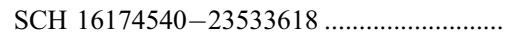 & 19.2 & 16.3 & 14.05 & 13.31 & 12.95 & 2.83 & 1.67 & 0.91 & M6 & -15.50 & USco \\
\hline 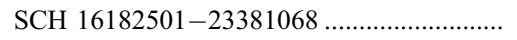 & 18.9 & 16.1 & 13.72 & 12.88 & 12.44 & 2.06 & 1.32 & 0.91 & M5 & -9.20 & USco \\
\hline 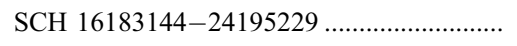 & 19.5 & 16.6 & 14.15 & 13.46 & 12.97 & 2.45 & 1.60 & 0.87 & M6.5 & -11.40 & Int \\
\hline 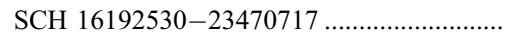 & 18.5 & 16.1 & 14.01 & 13.17 & 12.75 & 1.60 & 1.12 & 0.87 & M4 & $\ldots$ & Dwarf \\
\hline 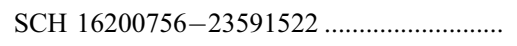 & 18.5 & 15.6 & 13.21 & 12.48 & 12.05 & 2.49 & 1.58 & 0.92 & M6 & -24.20 & USco \\
\hline 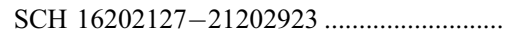 & 18.5 & 15.5 & 13.39 & 12.74 & 12.40 & 2.61 & 1.62 & 0.89 & M6 & -23.90 & USco \\
\hline 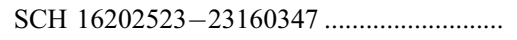 & 18.9 & 16.6 & 14.37 & 13.68 & 13.23 & 2.20 & 1.53 & 0.88 & M5.5 & -9.50 & USco \\
\hline SCH $16202753-14082840 \ldots \ldots \ldots \ldots \ldots \ldots \ldots \ldots$ & 16.0 & 13.8 & 12.22 & 11.56 & 11.27 & 1.85 & 1.20 & 0.84 & M4.5 & & Dwarf \\
\hline 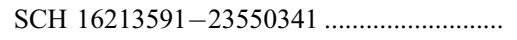 & 19.5 & 16.5 & 13.94 & 13.19 & 12.73 & 2.38 & 1.69 & 0.90 & M6 & -19.90 & USco \\
\hline 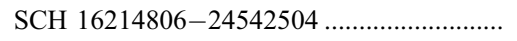 & 19.7 & 17.4 & 15.19 & 14.22 & 13.61 & 1.23 & 0.98 & 0.93 & M2 & $\ldots$ & Dwarf \\
\hline 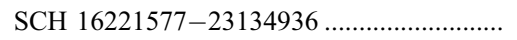 & 18.3 & 15.8 & 13.71 & 13.14 & 12.80 & 2.52 & 1.55 & 0.87 & M6 & -9.20 & Int \\
\hline 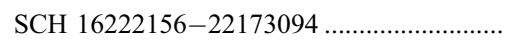 & 18.0 & 15.5 & 13.74 & 13.09 & 12.61 & 2.00 & 1.36 & 0.92 & M5 & -60.30 & USco \\
\hline 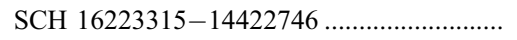 & 17.6 & 15.3 & 13.49 & 12.84 & 12.51 & 2.11 & 1.30 & 0.79 & M5 & $\ldots$ & Dwarf \\
\hline 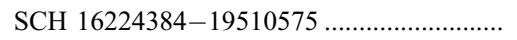 & 17.0 & 15.0 & 12.35 & 11.61 & 11.15 & 2.42 & 2.13 & 0.98 & M8 & -62.10 & USco \\
\hline 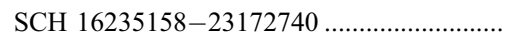 & 19.3 & 16.1 & 13.55 & 12.89 & 12.41 & 2.82 & 2.16 & 0.96 & M8 & -76.80 & USco \\
\hline 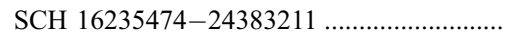 & 19.1 & 16.0 & 13.31 & 12.49 & 11.92 & 2.34 & 1.61 & 0.95 & M6 & -12.80 & USco \\
\hline 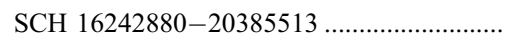 & 18.9 & 16.7 & 14.58 & 13.77 & 13.31 & 1.50 & 1.08 & 0.92 & M3 & $\ldots$ & Dwarf \\
\hline 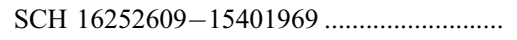 & 17.4 & 15.3 & 13.67 & 12.94 & 12.52 & 1.52 & 1.07 & 0.93 & M3 & $\ldots$ & Dwarf \\
\hline 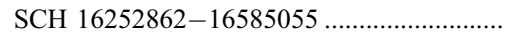 & 19.2 & 16.2 & 13.67 & 13.01 & 12.62 & 2.66 & 1.94 & 0.94 & M8 & -23.30 & USco \\
\hline 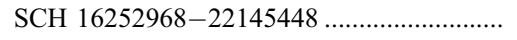 & 18.0 & 15.4 & 13.19 & 12.49 & 12.11 & 2.25 & 1.41 & 0.94 & M5 & -16.20 & USco \\
\hline 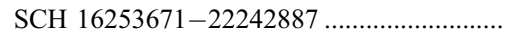 & 18.7 & 15.9 & 13.53 & 12.83 & 12.45 & 2.64 & 1.76 & 0.93 & M7 & -11.60 & USco \\
\hline 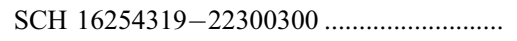 & 16.8 & 15.0 & 13.02 & 12.40 & 12.09 & 2.19 & 1.37 & 0.90 & M5 & -9.20 & USco \\
\hline 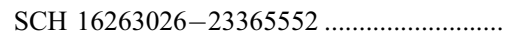 & 18.9 & 16.5 & 13.75 & 12.82 & 12.21 & 2.51 & 1.69 & 0.94 & M6 & -32.10 & USco \\
\hline 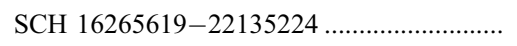 & 18.5 & 15.7 & 13.48 & 12.83 & 12.41 & 2.71 & 1.67 & 0.90 & M6 & -28.40 & USco \\
\hline 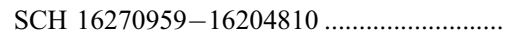 & 17.4 & 15.4 & 13.55 & 12.72 & 12.28 & 1.19 & 1.01 & 0.95 & M2 & $\ldots$ & Dwarf \\
\hline 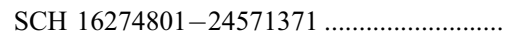 & 19.2 & 16.2 & 13.54 & 12.65 & 12.11 & 2.20 & 1.42 & 0.91 & M5 & -22.00 & USco \\
\hline 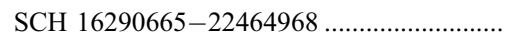 & 18.6 & 16.2 & 14.03 & 13.19 & 12.77 & 1.65 & 1.10 & 0.86 & M4 & -9.50 & Dwarf \\
\hline 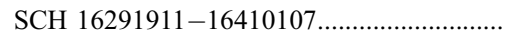 & 16.8 & 14.6 & 12.85 & 12.12 & 11.76 & 1.62 & 1.11 & 0.85 & M3.5 & -3.70 & Dwarf \\
\hline 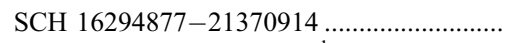 & 16.9 & 14.5 & 12.52 & 11.86 & 11.52 & 2.16 & 1.34 & 0.92 & M5 & -9.20 & USco \\
\hline SCH $16300911-24111718^{\mathrm{d}} \ldots \ldots \ldots \ldots \ldots \ldots \ldots \ldots$ & 16.1 & 14.7 & 13.38 & 12.84 & 12.56 & 1.48 & 1.05 & 0.87 & M2 & $\ldots$ & Dwarf \\
\hline 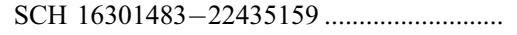 & 18.8 & 16.7 & 14.57 & 13.71 & 13.20 & 1.48 & 1.02 & 0.89 & M3 & ... & Dwarf \\
\hline
\end{tabular}


TABLE 1-Continued

\begin{tabular}{|c|c|c|c|c|c|c|c|c|c|c|c|}
\hline ID & $R$ & $I$ & $J^{\mathrm{a}}$ & $H^{\mathrm{a}}$ & $K_{S}^{\mathrm{a}}$ & TiO-7140 & TiO- 8465 & Na-8189 & Spectral Type ${ }^{b}$ & $\begin{array}{c}W(\mathrm{H} \alpha) \\
(\AA)\end{array}$ & Gravity $^{\mathrm{c}}$ \\
\hline SCH $16301682-15574807 \ldots$ & 18.0 & 15.6 & 13.59 & 12.86 & 12.53 & 1.95 & 1.14 & 0.81 & M5 & -4.30 & Dwarf \\
\hline 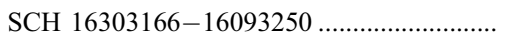 & 17.5 & 15.2 & 13.38 & 12.69 & 12.37 & 1.80 & 1.20 & 0.83 & M4 & -5.40 & Dwarf \\
\hline SCH 16324726-20593771 .. & 17.9 & 15.5 & 13.45 & 12.85 & 12.47 & 2.39 & 1.50 & 0.91 & M6 & -25.20 & USco \\
\hline 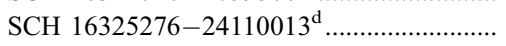 & 17.1 & 15.9 & 14.33 & 13.69 & 13.40 & 0.96 & 0.94 & 0.98 & $<\mathrm{K} 3$ & $\ldots$ & Dwarf \\
\hline SCH $16325602-16582835 \ldots \ldots \ldots \ldots \ldots \ldots \ldots$ & 15.9 & 13.7 & 12.26 & 11.76 & 11.44 & 1.94 & 1.19 & 0.84 & M4 & $\ldots$ & Dwarf \\
\hline
\end{tabular}

${ }^{\text {a }}$ Near-infrared photometry taken from 2MASS.

${ }^{\mathrm{b}}$ Spectral type errors are \pm 0.5 for $\mathrm{M}$ subclasses. A colon indicates that a spectral type is less certain.

" Qualitative surface gravity type "USco" and "dwarf" labels indicate that a star has surface gravity signatures consistent with those measured for known USco members or field dwarfs. A value of "int" corresponds to the object having a gravity signature between those of known USco members and field dwarfs.

d Photometry for three targets changed significantly after spectroscopic observations took place, such that they would no longer have been chosen as photometric candidates.

${ }^{\mathrm{e}}$ The only object among our spectral targets previously identified in the literature is SCH 16044303-23182620, corresponding to UScoCTIO110 (Ardila et al. 2000).

All sources, including standards, were preprocessed, extracted, and flux-calibrated using standard IRAF tasks.

\section{SPECTRAL ANALYSIS}

The 6300-8825 \& wavelength regime contains many temperature-sensitive and surface-gravity-sensitive features diagnostic for classifying late-type stars. To ensure that we could accurately classify our program stars, we observed a range of spectral main-sequence standards (K5-L1.5), giant standards (K7-M9), and previously identified USco objects (K3-M8; Preibisch \& Zinnecker 1999; Ardila et al. 2000; Martín et al. 2004). In addition, we took observations of spectroscopically confirmed 1 Myr Taurus members and 100 Myr Pleiades stars during an observing run in 2004 December with the same telescope and instrument setup. Together these observations provide a large range in both temperature and surface gravity, which we use for aid in classifying program stars.

In this section we use molecular absorption features to derive a spectral type for each object. From atomic and molecular surface gravity diagnostics we determine which objects have low PMS-type gravity and are therefore likely association members. The effects of reddening and veiling are explored and considered in both temperature and gravity determination.

\subsection{Temperature Classification}

Figure 8 shows spectra for dwarf stars of spectral types M3, M6, and M8. The dominant molecular absorption features present are attributed to titanium oxide ( $\mathrm{TiO}$ ), and the strongest of these are labeled. TiO absorption increases from mid-K through $\sim \mathrm{M} 7$ spectral types, at which point its strength begins to decrease and vanadium oxide ( $\mathrm{VO}$ ) absorption starts to dominate spectra by early L types. We have defined or adopted from the literature several band indices to measure the strength of the $\mathrm{TiO}$ absorption features. We find two in particular to be diagnostic. TiO-7140 measures the strength of TiO $\lambda 7140$ absorption compared to a continuum band at $7035 \AA$ (Wilking et al. 2005). This index is defined as TiO-7140 $=F_{\lambda 7035} / F_{\lambda 7140}$ with bandwidths of $50 \AA$. TiO-8465 is a new index defined by us (TiO-8465 = $\left.F_{\lambda 8415} / F_{\lambda 8465}\right)$ to measure the strength of TiO $\lambda 8465$ absorption compared to a continuum band at $8415 \AA$. Bandwidths for the TiO-8465 index are $20 \AA$. In Figure 8, light and dark shaded regions show, respectively, the location of the $\mathrm{TiO}$ and continuum bands used in our analysis.

Figure 9 (left) shows a plot of TiO-8465 versus TiO-7140; black spectral types are measured indices for field dwarf spectra, and blue spectral types are measured indices for known USco and Taurus members. This diagram is particularly useful for classifying objects with spectral types $\sim \mathrm{M} 3-\mathrm{L} 3$. We find no surfacegravity dependence between measured indices for the dwarf, USco, and Taurus standard objects. In Figure 9 (right), green circles correspond to measured indices for program stars. As can be seen, the program star measurements follow the locus laid out by the standard stars extremely well. We find six outliers that sit below the main locus of data points. In all six cases, the star is confirmed to exhibit low-gravity signatures $(\S 3.2$ ), and we attribute the position in Figure 9 to a small amount of veiling present in its spectra (§ 3.3). These objects are further discussed in $\S 4.2$.

Spectral types were determined first from quantitative analysis using an object's measured $\mathrm{TiO}$ indices. More weight was given to the value of the TiO-8465 index, which we find particularly insensitive to the effects of reddening and veiling, as discussed in $\S 3.3$. Using the $\mathrm{TiO}$ indices alone, we cannot uniquely recover spectral types for the dwarf and PMS standards. This ambiguity is due in part to uncertainties in published spectral types and in part to systematic differences between spectral classification schemes of different authors. Therefore, it was necessary to examine each spectrum by eye and use the information from the entire spectral range in the final type determination. All program spectra were compared visually to a large grid of

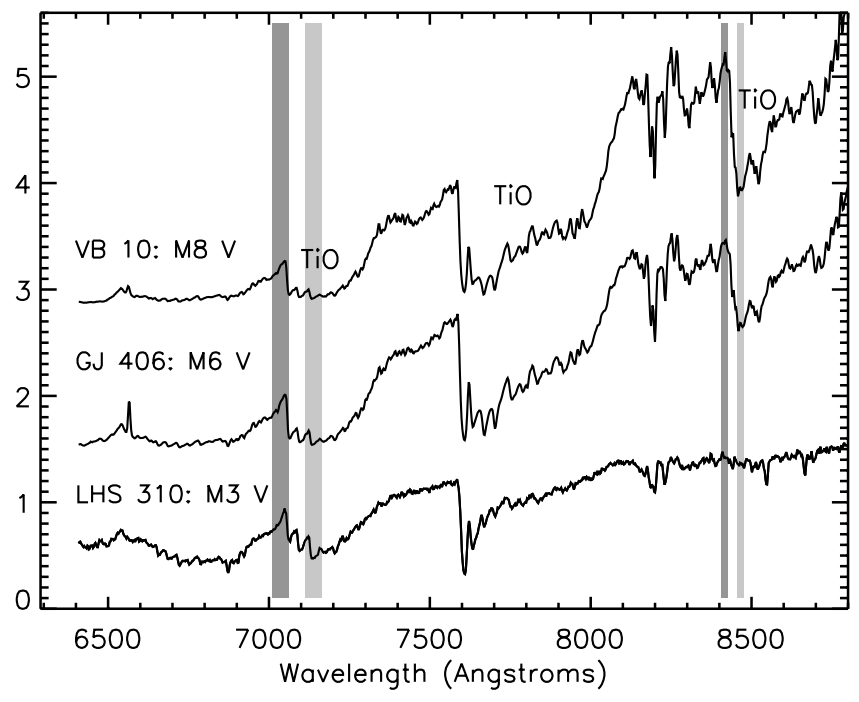

FIG. 8.- Spectra for M3-, M6-, and M8-type dwarf stars. Dominant TiO features are labeled. Light and dark shaded regions show, respectively, the location of the $\mathrm{TiO}$ and continuum bands used in spectral classification. 

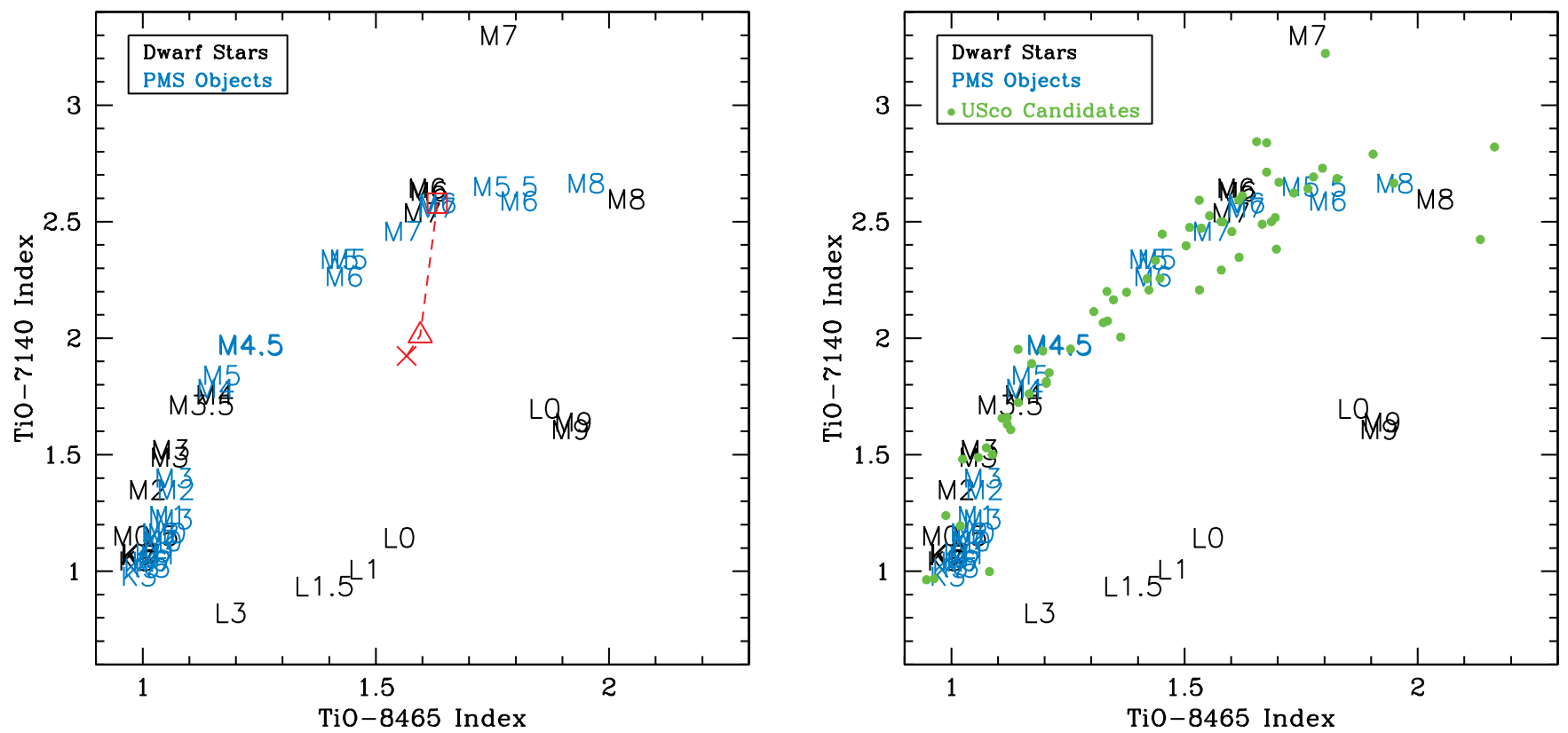

FIG. 9. - Left: Two temperature-sensitive indices, TiO- $\lambda 7140 \mathrm{vs.} \mathrm{TiO-8465.} \mathrm{Spectral} \mathrm{types} \mathrm{represent} \mathrm{measured} \mathrm{indices} \mathrm{for} \mathrm{dwarf} \mathrm{stars} \mathrm{(black)} \mathrm{and} \mathrm{known} \mathrm{USco} \mathrm{and} \mathrm{Taurus}$ members (blue). Right: Same as the left panel, but with green circles corresponding to measured indices for program stars. In the left panel, connected red symbols indicate the effects of veiling from a cool circumstellar disk (square) or a hot accretion shock (triangle and cross) on measured indices for a single star (see text for more details).

standard spectra. Typical revisions to the quantitative spectral types were at the level of 1-2 subclasses.

\subsection{Surface Gravity Assessment}

Accurate spectral classification requires determining both the type (indicative of temperature) and the luminosity class (indicative of gravity) of our candidates. Several robust surface-gravitysensitive features exist in the 6300-8800 A spectral regime that can be assessed in low-resolution spectra and used to distinguish high-gravity dwarf stars from younger PMS objects still undergoing contraction. The three most prominent features are due to Na I (8183 and $8195 \AA$ ) and K I (7665 and $7699 \AA)$ lines and $\mathrm{CaH}$ molecular absorption at $6975 \AA$. Figure 10 shows a sequence of three M6 standard stars: a high-gravity main-sequence star (GJ 406), a 5 Myr intermediate-gravity USco object (DENIS-P 16007.5-181056.4), and a 1 Myr lower gravity Taurus object (MH 05). Gravity-sensitive absorption features are labeled and increase in strength with increasing stellar age and gravity.

We have developed a gravity-sensitive index, Na-8189, which measures the strength of the Na I doublet at $8189 \AA$ compared to the strength of a continuum band at $8150 \AA$. Each band is $30 \AA$ wide. The index is defined as Na-8189 $=F_{\lambda 8189} / F_{\lambda 8150}$. Figure 11 (left) shows a plot of the temperature-sensitive TiO-7140 index versus the gravity-sensitive Na-8189 index. Black and blue spectral types, respectively, represent measured indices for dwarfs and known PMS stars, similar to Figure 9. Cyan spectral types show measured indices for low-gravity giant standard stars. We find the Na-8189 index to be a robust diagnostic that clearly separates low, intermediate, and high gravity for spectral types later than M2. Objects with spectral types earlier than $\sim \mathrm{M} 2$ do not exhibit substantial Na I absorption. Measured Na-8189 indices for 100 Myr Pleiades stars are indistinguishable from those for dwarfs at similar spectral types. In Figure 11 (right) green circles correspond to measured indices for program stars. A large fraction, 43/62, of the candidate objects have measured $\mathrm{Na}-8189$ indices consistent with their having surface gravity less than that of field dwarfs at similar spectral types.

Gravity signatures in all spectra were verified visually. For only one star did we find a visual assessment that disagreed with the quantitative result of the Na-8189 index. SCH 1622438419510575 appears to be a giant star based on the $\mathrm{Na}-8189$ index alone but was determined from visual inspection to be a young PMS-type object based on the overall shape of its spectrum longward of $\sim 8200 \AA$ and strong $\mathrm{H} \alpha$ emission ( $\S 3.4)$. This object is discussed further in $\S 4.2$.

\subsection{Stellar and Interstellar Processes That Can Affect Spectral Classification}

In this section we explore possible biases in the spectral classification due to the effects of stellar and interstellar reddening or spectral veiling produced either from a cool circumstellar disk or

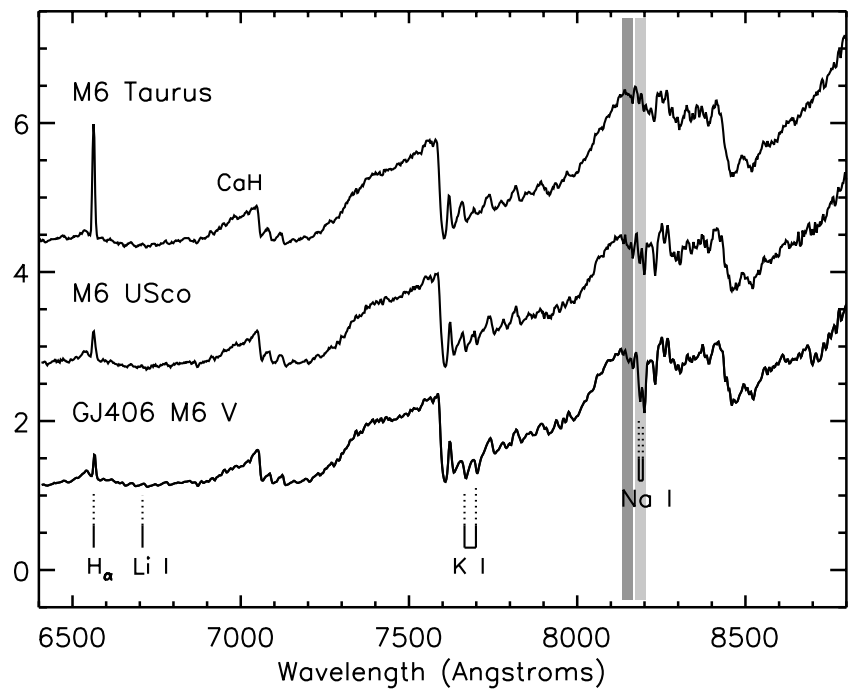

FIG. 10.- Sequence of three M6 stars: a high-gravity main-sequence star (GJ 406), a 5 Myr intermediate-gravity USco object (DENIS-P 16007.5-181056.4), and a 1 Myr Taurus object (MH 05). Gravity-sensitive absorption features are labeled and increase with increasing stellar age and gravity. Light and dark shaded regions show, respectively, the locations of the $\mathrm{Na}$ and continuum bands used in surface gravity analysis. 

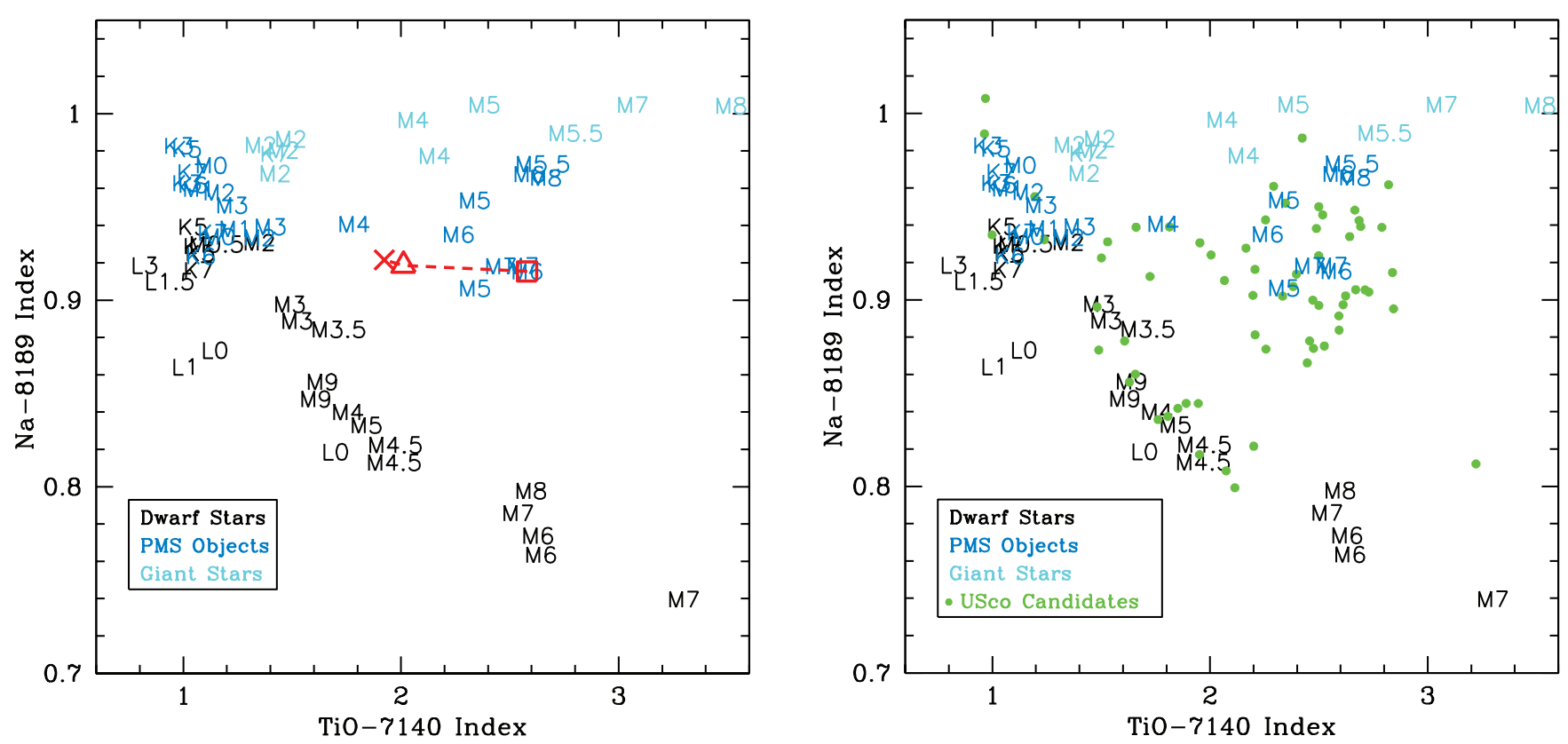

FIg. 11.-Same as Fig. 9, but for the TiO-7140 index vs. the gravity-sensitive Na-8189 index. Cyan spectral types represent measured indices for low-gravity giant stars.

from a hot accretion shock. We assess the effects these processes can have both on the quantitative $\mathrm{TiO}$ and $\mathrm{Na}$ indices and on the overall appearance of a spectrum.

\subsubsection{Extinction}

Hot OB stars have dispersed much of the dense gas and dust in the USco region; therefore, we do not expect individual members to show more than moderate extinction. We derived an approximate $A_{V}$ measurement for each star by visually dereddening its spectrum until its slope matched that of a standard star of the same spectral type. We find that $80 \%$ of the newly identified members have $A_{V}<1$ based on spectral slope from 6300 to $8825 \AA$, and only three appear to have $A_{V}$ between 2 and 3 mag. More precise extinction values are derived from each object's spectral type and colors in $\S 4.1$, and in all cases we find agreement to within 1 mag of our visual estimate.

To assess the effect reddening has on our quantitative classification indices we artificially reddened all standard stars by $A_{V}=$ $10 \mathrm{mag}$, the maximum extinction inferred from large-beam dust measurements toward our survey region excluding the young $\rho$ Ophiuchus molecular cloud core (Schlegel et al. 1998). We measured index strengths for artificially reddened spectra and found average index shifts of -0.1 for $\mathrm{TiO}-7140$ and -0.04 for TiO8465. As can be seen from Figure 9, index shifts at this level are not sufficient to affect our quantitative temperature determination from $\mathrm{TiO}$ indices at the 0.5 subclass level. Ten mag of extinction could affect the $\mathrm{Na}-8189$ index by an average shift of -0.05 . However, at a level of reddening more consistent with actual measured values for new members $\left(A_{V} \lesssim 2 ; \S 4.1\right.$, Table 2$)$, we find a much more moderate shift of -0.01 , which would not be sufficient to make a dwarf star look like a PMS star for objects between M2 and M9.

\subsubsection{Optical and Near-Infrared Veiling}

A young star spectrum can be veiled at UV/optical wavelengths due to excess emission from an accretion shock, and in the infrared due to thermal emission from dust grains in a circumstellar disk. In both cases the excess emission veils (decreases) the strength of the molecular absorption features used in classification and causes a star to be systematically classified too hot (early) in spectral type. The veiling index is defined as $r_{\lambda}=F_{\lambda \mathrm{ex}} / F_{\lambda \mathrm{ph}}$, where "ex" indicates excess and "ph" indicates photosphere. We tested several scenarios to determine the existence and magnitude of any bias in the spectral types attributable to veiling.

First, we added to all spectra a $T_{\text {eff }}=1400 \mathrm{~K}$ blackbody consistent with a cool disk around a low-mass star or brown dwarf at a veiling level of $r_{K}=0.6$, corresponding to the median nearinfrared veiling value for K7-M0 classical T Tauri stars (Meyer et al. 1997). The Wein tail of such a blackbody could affect our spectral indices around $0.8 \mu \mathrm{m}$. We remeasured indices for veiled standard spectra and found, as expected, that excess thermal emission from a cool disk has very little effect on optical spectra. Results from veiling experiments are shown for a single star (DENIS-P 16007.5-181056.4, an M6 USco member; Martín et al. 2004) in Figures 9 and 11 (left panels, connected red symbols). We see similar results for all M-type (spectral type $>\mathrm{M} 2$ ) standards artificially veiled using these techniques. The rederived indices for DENIS-P 16007.5-181056.4 plus near-infrared veiling described above are shown as red squares that lie practically on top of the original "M6" blue points in both figures.

Next we investigated the effects of veiling from a hot accretion shock. We used a value of $r_{6500}=0.6$, equivalent to the average optical veiling value for late $\mathrm{K}$ and early $\mathrm{M}$ stars (White \& Hillenbrand 2004). We added a $T_{\text {eff }}=8000 \mathrm{~K}$ blackbody at this level to all standard spectra and rederived the spectral classification indices (Figs. 9 and 11, triangles). We experimented by adding a continuum excess of constant flux $\left(F_{\mathrm{ex}}=C\right)$ at the $r_{6500}=$ 0.6 level (Figs. 9 and 11, crosses), shown by White \& Hillenbrand (2004) to be more consistent with observations than a hot blackbody. In both cases, the strength of the TiO-7140 index decreases substantially, while the changes in the TiO-8465 and Na-8189 indices are much smaller. For this reason we rely primarily on the TiO-8465 index for temperature classification and believe our surface gravity assessment from the Na-8189 index to be robust to effects of veiling. 
TABLE 2

Derived Quantities for New USco Members

\begin{tabular}{|c|c|c|c|c|}
\hline ID & $M_{J}$ & $A_{V}$ & $\log \left(T_{\text {eff }} / \mathrm{K}\right)$ & $\log \left(L / L_{\odot}\right)$ \\
\hline SCH $15583162-24025411 \ldots \ldots \ldots$. & 7.18 & 0.66 & 3.48 & -1.76 \\
\hline SCH $15594802-22271650 \ldots \ldots \ldots$ & 8.35 & 0.33 & 3.41 & -2.27 \\
\hline SCH $16014768-24410152 \ldots \ldots \ldots$ & 8.03 & 0.15 & 3.47 & -2.11 \\
\hline SCH $16040453-23463795 \ldots \ldots \ldots$. & 5.64 & 1.14 & 3.49 & -1.13 \\
\hline SCH $16044303-23182620 \ldots \ldots \ldots$ & 7.99 & 0.05 & 3.43 & -2.12 \\
\hline SCH $16051829-17562092 \ldots \ldots \ldots$. & 5.62 & 0.85 & 3.49 & -1.12 \\
\hline SCH $16053077-22462016 \ldots \ldots \ldots$ & 7.97 & 0.04 & 3.44 & -2.11 \\
\hline SCH $16075850-20394890 \ldots \ldots \ldots$. & 7.69 & 0.38 & 3.44 & -1.99 \\
\hline SCH $16093018-20595409 \ldots \ldots \ldots$ & 8.09 & 0.39 & 3.44 & -2.15 \\
\hline SCH $16095991-21554293 \ldots \ldots \ldots$ & 8.38 & 0.43 & 3.43 & -2.28 \\
\hline SCH $16103876-18292353 \ldots \ldots \ldots$ & 7.69 & 1.78 & 3.44 & -1.99 \\
\hline SCH $16111711-22171749 \ldots \ldots \ldots . . .$. & 8.54 & 0.00 & 3.41 & -2.35 \\
\hline SCH $16112959-19002921 \ldots \ldots \ldots$. & 7.46 & 1.52 & 3.44 & -1.90 \\
\hline SCH $16121188-20472698 \ldots \ldots \ldots$. & 7.79 & 0.24 & 3.43 & -2.04 \\
\hline SCH $16123758-23492340 \ldots \ldots \ldots$ & 8.00 & 0.48 & 3.44 & -2.12 \\
\hline SCH $16124692-23384086 \ldots \ldots \ldots$. & 7.77 & 0.29 & 3.44 & -2.02 \\
\hline SCH $16131212-23050329 \ldots \ldots \ldots$ & 8.25 & 0.00 & 3.43 & -2.22 \\
\hline SCH $16132576-17373542 \ldots \ldots \ldots$. & 6.38 & 0.52 & 3.49 & -1.43 \\
\hline SCH $16141974-24284053 \ldots \ldots \ldots$. & 7.87 & 0.54 & 3.44 & -2.06 \\
\hline SCH $16151115-24201556 \ldots \ldots \ldots . .$. & 8.32 & 0.42 & 3.44 & -2.24 \\
\hline SCH $16155508-24443677 \ldots \ldots \ldots$. & 7.46 & 0.49 & 3.44 & -1.90 \\
\hline SCH $16172504-23503799 \ldots \ldots \ldots$ & 7.59 & 1.31 & 3.47 & -1.93 \\
\hline SCH $16174540-23533618 \ldots \ldots \ldots$. & 7.91 & 1.26 & 3.44 & -2.08 \\
\hline SCH $16182501-23381068 \ldots \ldots \ldots$ & 7.29 & 2.37 & 3.47 & -1.82 \\
\hline SCH $16183144-24195229 \ldots \ldots \ldots$. & 8.15 & 0.76 & 3.43 & -2.18 \\
\hline SCH $16200756-23591522 \ldots \ldots \ldots$. & 7.09 & 1.18 & 3.44 & -1.75 \\
\hline SCH $16202127-21202923 \ldots \ldots \ldots$. & 7.48 & 0.41 & 3.44 & -1.91 \\
\hline SCH $16202523-23160347 \ldots \ldots \ldots$ & 8.31 & 0.95 & 3.45 & -2.23 \\
\hline SCH $16213591-23550341 \ldots \ldots \ldots$ & 7.78 & 1.38 & 3.44 & -2.03 \\
\hline SCH $16221577-23134936 \ldots \ldots \ldots$. & 7.91 & 0.00 & 3.44 & -2.08 \\
\hline SCH $16222156-22173094 \ldots \ldots \ldots$. & 7.76 & 0.66 & 3.47 & -2.00 \\
\hline SCH $16224384-19510575 \ldots \ldots \ldots .$. & 6.35 & 0.75 & 3.40 & -1.48 \\
\hline SCH $16235158-23172740 \ldots \ldots \ldots$ & 7.75 & 0.00 & 3.40 & -2.04 \\
\hline SCH $16235474-24383211 \ldots \ldots \ldots$ & 6.96 & 2.07 & 3.44 & -1.70 \\
\hline SCH $16252862-16585055 \ldots \ldots \ldots$ & 7.86 & 0.02 & 3.40 & -2.08 \\
\hline SCH $16252968-22145448 \ldots \ldots \ldots$. & 7.09 & 1.14 & 3.47 & -1.73 \\
\hline SCH $16253671-22242887 \ldots \ldots \ldots$. & 7.55 & 0.67 & 3.42 & -1.95 \\
\hline SCH $16254319-22300300 \ldots \ldots \ldots$ & 7.12 & 0.38 & 3.47 & -1.74 \\
\hline SCH $16263026-23365552 \ldots \ldots \ldots$ & 7.15 & 3.02 & 3.44 & -1.78 \\
\hline SCH $16265619-22135224 \ldots \ldots \ldots$. & 7.57 & 0.39 & 3.44 & -1.94 \\
\hline SCH $16274801-24571371 \ldots \ldots \ldots$ & 6.99 & 2.82 & 3.47 & -1.70 \\
\hline SCH $16294877-21370914 \ldots \ldots \ldots$. & 6.53 & 0.73 & 3.47 & -1.51 \\
\hline SCH $16324726-20593771 \ldots \ldots \ldots$ & 7.64 & 0.06 & 3.44 & -1.97 \\
\hline
\end{tabular}

\subsection{Emission Lines}

The only prominent emission line observed in any of the spectra is $\mathrm{H} \alpha$, which, seen in the spectra of young stars and brown dwarfs, is predominantly created via one of two mechanisms. Weak, narrow $\mathrm{H} \alpha$ lines are presumed to originate from active chromospheres, whereas strong, broad and/or asymmetric lines can be produced from high-velocity infalling accretion or strong winds. Barrado y Navascués \& Martìn (2003) have proposed an empirical spectral type- $\mathrm{H} \alpha$ equivalent width $[W(\mathrm{H} \alpha)]$ relation to describe the upper limit of nonaccreting stars and brown dwarfs based on the chromospheric saturation limit observed in young open clusters. Figure 12 plots measured $\mathrm{H} \alpha$ equivalent widths for all spectra as a function of spectral type, shown with the Barrado y Navascués \& Martin (2003) empirical accretor/nonaccretor division. Four objects (SCH 16222156-22173094: M5; SCH 16103876-18292353: M6; SCH 16224384-19510575: M8; SCH 16235158-23172740: M8; see Table 1) exhibit very strong emission $[W(\mathrm{H} \alpha)<-60 \AA]$ at levels substantially above those

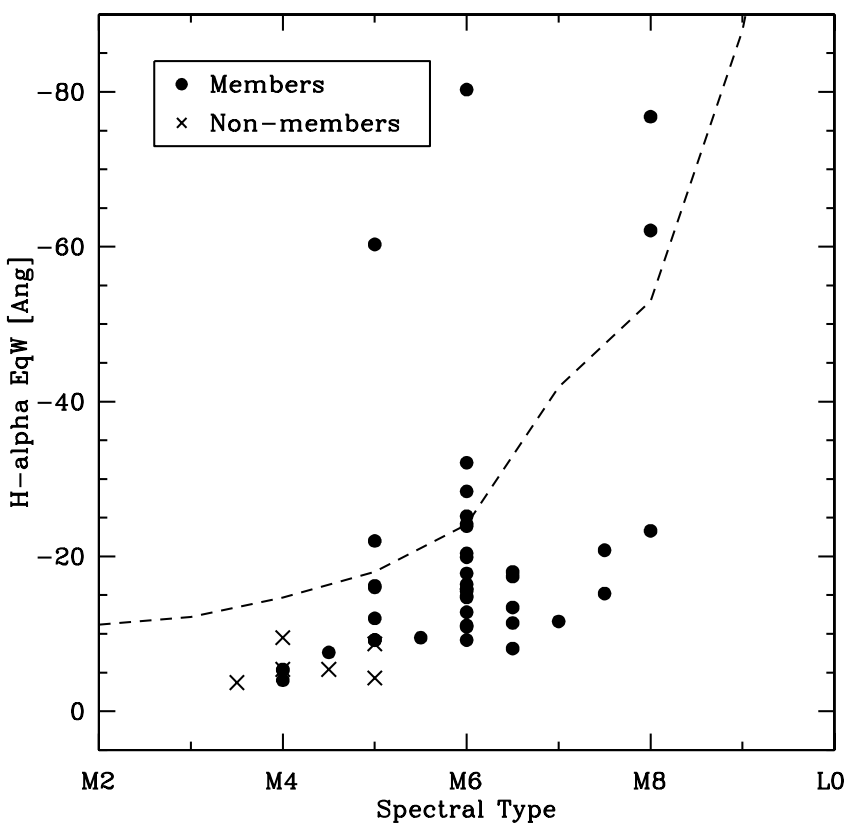

FIG. 12.-Measured $\mathrm{H} \alpha$ equivalent widths for all spectra with measurable $\mathrm{H} \alpha$ as a function of spectral type. Circles represent objects confirmed to be new members; crosses represent objects confirmed to be field dwarfs. The dotted line is the empirical accretor/nonaccretor upper limit derived by Barrado y Navascués \& Martín (2003).

measured for the majority of sources in our sample and are possibly still undergoing active accretion. High-resolution spectroscopy is needed to assess further the evidence for accretion based on line profiles/shape.

\subsection{Summary of Spectral Classification and Analysis}

We have determined spectral types and surface gravity estimates for 62 objects toward the USco association. These objects were selected as candidate PMS association members based on their optical and near-infrared colors and magnitudes. In all cases classification was done first using flux ratios of broad molecular absorption lines or narrow atomic lines to continuum levels. Reddening and veiling were accounted for during the spectral classification and gravity assessment processes. All spectral types and surface gravity estimates were confirmed from visual comparison to standard spectra. Unless otherwise noted in Table 1, spectral type errors are \pm 0.5 .

Table 1 lists optical and near-infrared 2MASS photometry, measured spectral indices, spectral types, and $\mathrm{H} \alpha$ equivalent widths. We also list a qualitative surface gravity type: "USco" and "dwarf" labels indicate a star that has surface gravity signatures consistent with those measured for known USco members or field dwarfs, respectively. A value of "int" corresponds to the object having gravity signatures between those of known USco members and field dwarfs, indicating that it is likely a more evolved association member, a member of one of the neighboring Sco-Cen subgroups, or a very young field object. As mentioned previously, we find Na-8189 indices for Pleiades objects to be indistinguishable from those measured for dwarf stars. Therefore the "int" objects are most probably between 5 and $\sim 100$ Myr old. For the remainder of this paper we consider all objects with gravity classification "USco" or "int" as new association members.

\section{USco POPULATION ANALYSIS}

\subsection{H-R Diagram}

In this section we combine each new member's spectral type and photometry to derive values for its luminosity and effective 


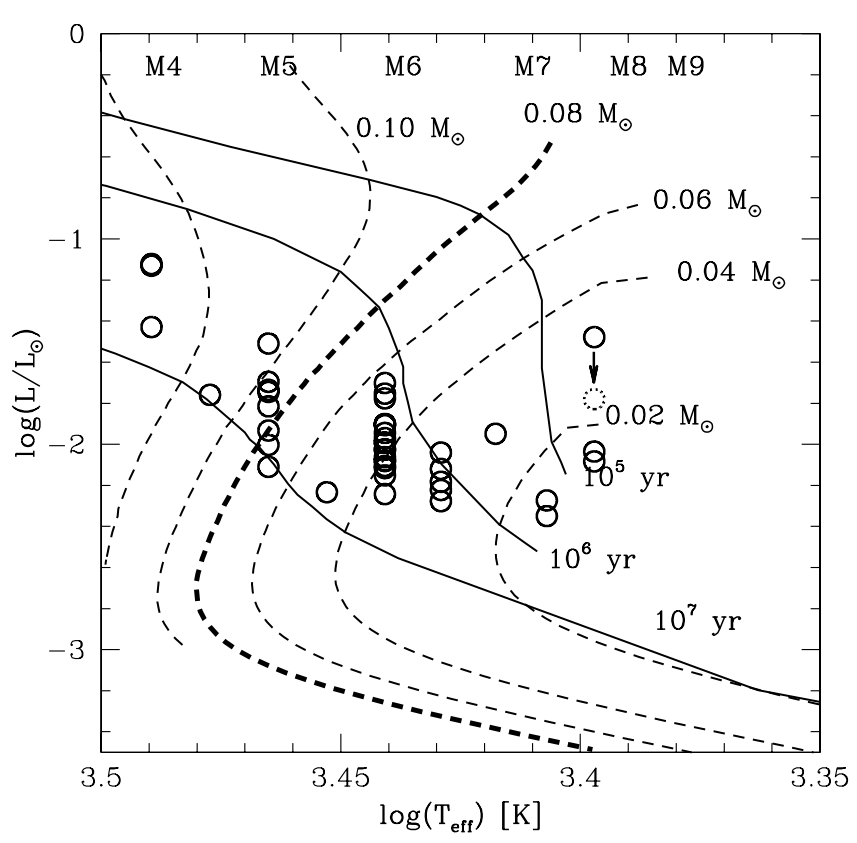

Fig. 13.- H-R diagram for new PMS objects found in this work, shown with model tracks and isochrones of DM97. The sample is consistent with an age of $\sim 5 \mathrm{Myr}$ and contains masses spanning the brown-dwarf to stellar regimes.

temperature and place it on a theoretical H-R diagram. As described in $\S 2$, the final calibration of Quest-2 optical data is still under revision. Thus, because of the reliability and uniformity of the 2MASS survey we chose to use $J$-band magnitudes and $(J-H)$ colors to derive luminosities. An empirical fit to $\mathrm{BC}_{J}$ as a function of spectral type was determined from the observational data of Leggett et al. (1996, 2002; spectral types M1-M6.5 and M6-L3). We adopted intrinsic colors, extinction, and effective temperatures using the methods described in Slesnick et al. (2004). Derived quantities are given in Table 2.

In Figure 13 we present an H-R diagram for the 43 newly identified low-mass members of USco, shown with the PMS model tracks and isochrones of DM97. The most commonly used PMS models for low-mass stars and brown dwarfs are those derived by DM97 and Baraffe et al. (1998), which differ primarily in their atmospheric approximations and treatment of convection. No models to date have consistently reproduced dynamical masses for young low-mass objects $\left(M<1.2 M_{\odot}\right.$; Hillenbrand \& White 2004). We therefore do not attempt to derive masses and ages for new members at this time. Both models suggest similar mass ranges for our data of $0.02 M_{\odot}<M<$ $0.2 M_{\odot}$, although predicted masses for individual objects can vary by up to $0.07 M_{\odot}$. As illustrated in Figure 13, we have identified a low-mass stellar population of age roughly consistent with the $5 \mathrm{Myr}$ age inferred in previous work on the intermediatemass $\left(6 M_{\odot}<M<0.1 M_{\odot}\right)$ members of USco (Preibisch et al. 2002).

\subsection{Interesting Objects}

In this section we discuss objects and empirical observations worthy of comment. The one outlier to the main locus of USco points in Figure 13 is object SCH 16224384-19510575, which appears overluminous and extremely young compared to the other sources. This object also has strong $\mathrm{H} \alpha$ emission (see $\S 3.4$ ) with a slightly asymmetric profile. It is unlikely that this object is a single, extremely young $(<100,000 \mathrm{yr}$, based on H-R diagram placement) association member. The object could be a young

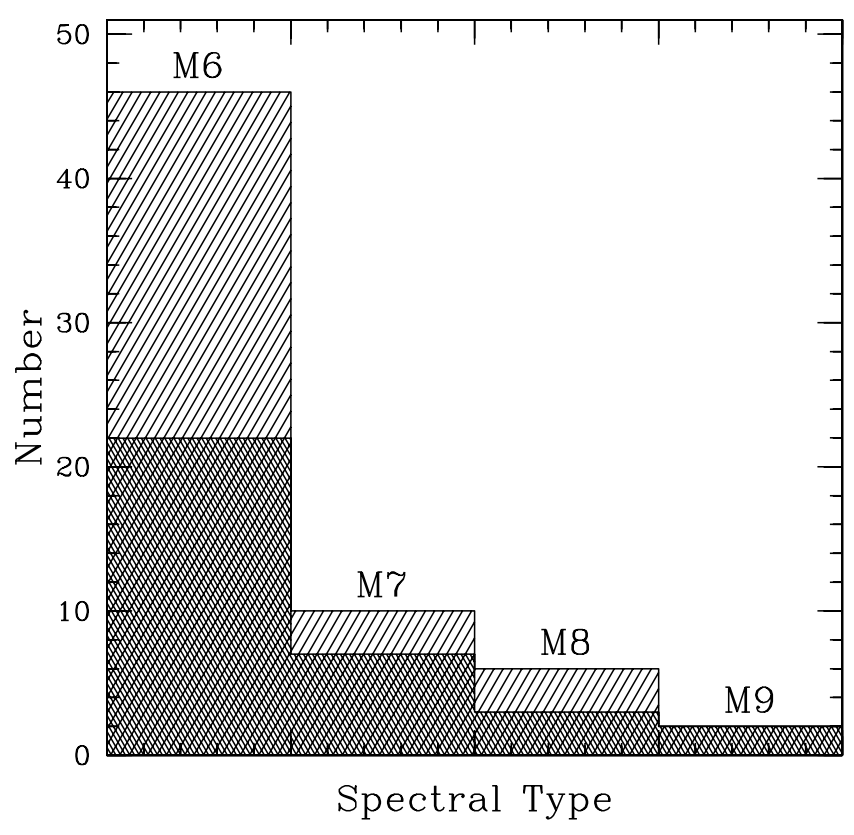

FIG. 14.-Histogram of the number of brown dwarfs spectroscopically confirmed in USco with the addition of our work (light shading) compared to a compilation of all previous results (dark shading).

PMS-gravity foreground object that happens to fall within our line of sight. The simplest explanation is that SCH 1622438419510575 is an unresolved binary. Assuming typical seeing at Palomar under photometric conditions of $\sim 1$.'2, any pair with separation $\leqslant 175 \mathrm{AU}$ would not be resolved in our data. If we assume SCH 16224384-19510575 consists of two equal-luminosity objects, its placement in the H-R diagram becomes more consistent with the main locus of association members. This effect is illustrated as an arrow and dotted circle in Figure 13.

The six objects with measured $\mathrm{TiO}$ indices below the main locus of points (see Fig. 9) are SCH 16103876-18292353 (M6), SCH 16202523-23160347 (M5.5), SCH 16213591-23550341 (M6), SCH 16222156-22173094 (M5), SCH 1622438419510575 (M8), and SCH 16235474-24383211 (M6). Three of these objects, one of which is the possible binary discussed above, have very strong $\mathrm{H} \alpha$ emission and are possible accretors. Three lie very close (within $\sim 1^{\circ}$ ) to the young $\rho$ Oph molecular cloud. However, because $\rho$ Oph and USco lie at approximately the same distance, if they were escaped $\leqslant 1$ Myr $\rho$ Oph members we would expect to see them exhibit systematically higher luminosities than USco members of similar spectral type. Based on Figure 13, this phenomenon is not observed. All six have a near-infrared excess based on $J-H, H-K_{S}$ colors (Fig. 6; Table 1), indicating the presence of an inner circumstellar disk. In every case we find spectral shapes consistent with a small amount of veiling $\left(r_{\lambda} \sim 0.1-\right.$ $0.2)$ in their spectra.

The spatial distribution of the four strong $\mathrm{H} \alpha$ emitters (possibly accreting objects; Fig. 1, boxes) follows a ridge of dust outlined by $100 \mu \mathrm{m} I R A S$ emission at the western edge of the association. All four objects are located outside of previous surveys that searched for low-mass members, and outside most of the known high-mass association members (Fig. 1, cyan plus signs). In general, we find some evidence for a trend of increased luminosity (within a given spectral type) for objects in the western portion of the cloud, independent of declination. Further spectroscopic observations will determine whether there exists a substantial population of young objects in these regions. 


\section{SUMMARY AND FUTURE WORK}

We have completed a large-area $R, I$ photometric survey in and near the Upper Scorpius region of recent star formation. From these data we selected candidate new PMS association members based on their optical and near-infrared colors and magnitudes. We present here results from the first effort in our spectroscopic follow-up campaign. We observed 62 candidates and determined $43(70 \%)$ to be bona fide new Upper Scorpius members. We derive an $\mathrm{H}-\mathrm{R}$ diagram for new members, mention noteworthy individual objects, and speculate on the spatial distribution of yet undiscovered low-mass association members.

At an age of $5 \mathrm{Myr}$, all objects with spectral type $\geq \mathrm{M} 6$ are commonly considered to be substellar. Based on this criterion, from the 43 new members we identify 30 new brown dwarfs. Prior to this work, 34 spectroscopically confirmed USco members had been identified at these spectral types (Preibisch \& Zinnecker 1999; Ardila et al. 2000; Martìn et al. 2004). In Figure 14 we present a histogram of the number of brown dwarfs known with the addition of our work (light shading) compared to what was known previously in the literature (dark shading). As can be seen, with this study we have doubled the number of known substellar objects in Upper Scorpius.

In addition to the data presented here, we have taken spectra of $\sim 1000$ candidates with the CTIO Hydra, which will be presented in a forthcoming paper. We have Spitzer Space Telescope IRAC data and approved Spitzer MIPS $24 \mu \mathrm{m}$ observations of 28 of the newly identified brown dwarfs, which we analyze in a forthcoming paper by Slesnick et al.

The authors would like to thank the entire Quest-2 collaboration and in particular David Rabinowitz, Anne Bauer, Jonathan Jerke, and Adam Rengstorf for observing and processing the photometric drift-scan data. We are appreciative to Ashish Mahabal and Milan Bogosavljevic for their help in understanding the Quest-2 systematics. We thank Russel White and David Ardila for discussions and insights that helped in our analysis.
Andrews, P. A. 2003, Ph.D. thesis, Yale Univ.

Ardila, D., Martín, E. L., \& Basri, G. 2000, AJ, 120, 479

Baraffe, I., Chabrier, G., Allard, F., \& Hauschildt, P. H. 1998, A\&A, 337, 403

Barrado y Navascués, D., \& Martín, E. L. 2003, AJ, 126, 2997

Cutri, R. M., et al. 2003, 2MASS All-Sky Catalog of Point Sources (Pasadena: IPAC)

D’Antona, F., \& Mazzitelli, I. 1997, Mem. Soc. Astron. Italiana, 68, 807 (DM97)

Desch, S., \& Ouellette, N. 2005, in Protostars and Planets V, ed. V. Manning et al. (Houston: Lunar and Planetary Institute), 8467

de Zeeuw, P. T., Hoogerwerf, R., de Bruijne, J. H. J., Brown, A. G. A., \& Blaauw, A. 1999, AJ, 117, 354

Feigelson, E. D., \& Decampli, W. M. 1981, ApJ, 243, 89

Fernie, J. D. 1983, PASP, 95, 782

Fukugita, M., Ichikawa, T., Gunn, J. E., Doi, M., Shimasaku, K., \& Schneider, D. P. 1996, AJ, 111, 1748

Haisch, K. E., Lada, E. A., \& Lada, C. J. 2001, ApJ, 553, L153

Hillenbrand, L. A. 2006, in A Decade of Discovery: Planets around Other Stars, ed. M. Livio (Baltimore: STScI), in press

Hillenbrand, L. A., \& White, R. J. 2004, ApJ, 604, 741

Kenyon, S. J., \& Hartmann, L. 1995, ApJS, 101, 117

Ku, W. H.-M., \& Chanan, G. A. 1979, ApJ, 234, L59

Leggett, S. K., Allard, F., Berriman, G., Dahn, C. C., \& Hauschildt, P. H. 1996, ApJS, 104, 117

Leggett, S. K., et al. 2002, ApJ, 564, 452

\section{REFERENCES}

Mamajek, E. E., et al. 2004, ApJ, 612, 496

Martín, E. L., Delfosse, X., \& Guieu, S. 2004, AJ, 127, 449

Massey, P., Strobel, K., Barnes, J. B., \& Anderson, E. 1988, ApJ, 328, 315

Meyer, M. R., Calvet, N., \& Hillenbrand, L. A. 1997, AJ, 114, 288

Parrao, L., \& Schuster, W. J. 2003, Rev. Mex. AA, 19, 81

Podosek, F., \& Cassen, P. 1994, Meteoritics, 29, 6

Preibisch, T., Brown, A., Bridges, T., Guenther, E., \& Zinnecker, H. 2002, AJ, 124, 404

Preibisch, T., Guenther, E., \& Zinnecker, H. 2001, AJ, 121, 1040

Preibisch, T., Guenther, E., Zinnecker, H., Sterzik, M., Frink, S., \& Roeser, S. 1998, A\&A, 333, 619

Preibisch, T., \& Zinnecker, H. 1999, AJ, 117, 2381

Preibisch, T., et al. 2005, ApJS, 160, 401

Rabinowitz, D., et al. 2003, BAAS, 203, 38.12

Schlegel, D. J., Finkbeiner, D. P., \& Davis, M. 1998, ApJ, 500, 525

Silverstone, M. D., et al. 2006, ApJ, 639, 1138

Skrutskie, M. F., et al. 1990, AJ, 99, 1187

Slesnick, C. L., Hillenbrand, L. A., \& Carpenter, J. M. 2004, ApJ, 610, 1045

Tachibana, S., \& Huss, G. R. 2003, AJ, 588, L41

Walter, F. M., Vrba, F. J., Mathieu, R. D., Brown, A., \& Myers, P. C. 1994, AJ, 107,692

White, R. J., \& Hillenbrand, L. A. 2004, ApJ, 616, 998

Wilking, B. A., Meyer, M. R., Robinson, J. G., \& Greene, T. P. 2005, AJ, 130, 1733 